\title{
Three-dimensional morphometric analysis reveals time-dependent structural changes in microglia and astrocytes in the central amygdala and hypothalamic paraventricular nucleus of heart failure rats
}

Ferdinand Althammer

Georgia State University

Hildebrando Candido Ferreira-Neto

Georgia State University

Myurajan Rubaharan

Georgia State University

\section{Ranjan Kumer Roy}

Georgia State University

javier E Stern ( $\boldsymbol{D}$ jstern@gsu.edu )

Georgia State University

\section{Research}

Keywords: hypothalamus, amygdala, microglia, astrocytes, A1, behavior, cytokines, heart failure, neuroinflammation, autonomic.

Posted Date: April 20th, 2020

DOI: https://doi.org/10.21203/rs.3.rs-22630/v1

License: (1) This work is licensed under a Creative Commons Attribution 4.0 International License. Read Full License

Version of Record: A version of this preprint was published on July 23rd, 2020. See the published version at https://doi.org/10.1186/s12974-020-01892-4. 


\section{Abstract}

Background Cardiovascular diseases, including heart failure are the most common cause of death globally. Recent studies support a high degree of comorbidity between heart failure and cognitive and mood disorders resulting in memory loss, depression and anxiety. While neuroinflammation in the hypothalamic paraventricular nucleus contributes to autonomic and cardiovascular dysregulation in heart failure, mechanisms underlying cognitive and mood disorders in this disease remain elusive. The goal of this study was to quantitatively asses markers of neuroinflammation (glial morphology, cytokines and $\mathrm{A} 1$ astrocyte markers) in the central amygdala, a critical forebrain region involved in emotion and cognition, and to determine its time course and correlation to disease severity during the progression of heart failure.

Methods We developed and implemented a comprehensive microglial/astrocyte profiler for precise threedimensional morphometric analysis of individual microglia and astrocytes in specific brain nuclei at different time points during the progression of heart failure. To this end, we used a well-established ischemic heart failure rat model. Morphometric studies were complemented with quantification of various pro-inflammatory cytokines and A1/A2 astrocyte markers via QPCR.

Results We report structural remodeling of central amygdala microglia and astrocytes during heart failure that affected cell volume, surface area, filament length and microglial branches, resulting overall in somatic swelling and deramification, indicative of a change in microglial state. These changes occurred in a time-dependent manner, correlated with the severity of heart failure, and were delayed compared to changes in the hypothalamic paraventricular nucleus. Morphometric changes correlated with elevated mRNA levels of pro-inflammatory cytokines and markers of reactive A1-type astrocytes in the paraventricular nucleus and central amygdala during heart failure.

Conclusion We provide evidence that in addition to the previously described hypothalamic neuroinflammation implicated in sympathohumoral activation during heart failure, microglia and astrocytes within the central amygdala also undergo structural remodeling indicative of glial shifts towards activated and pro-inflammatory phenotypes, respectively. Thus, our studies suggest that neuroinflammation in the amygdala stands as a novel pathophysiological mechanism and potential therapeutic target for that could be associated with emotional and cognitive deficits commonly observed at later stages during the course of heart failure.

\section{Background}

Cardiovascular diseases are the major cause of death globally, with 17.9 million people dying from CVDs in 2016, thereby representing $31 \%$ of all global deaths (World Health Organization, 2017). The vast majority of cardiovascular disease-related deaths (85\%) can be attributed to stroke and heart failure (HF) with their accompanying detrimental effects on the human body, albeit there might be substantial geographic differences in survivability rates (1). A growing body of clinical studies suggests a high 
degree of comorbidity between cardiovascular diseases and cognitive impairments and emotional distress (2-4). In fact, $20-40 \%$ of all HF patients develop major depression and display anxiety levels significantly higher than the healthy population $(2,3,5,6)$, which usually appear later than the cardiovascular and autonomic-related symptoms (7). However, the precise underlying neuronal substrates and mechanisms leading to mood disorders and cognitive impairments after HF remain largely unknown. Using the rodent left coronary ligation HF model, studies demonstrated that HF mice and rats exhibited behavioral changes including increased anxiety, depression, social impairments and anhedonia (8-10).

The paraventricular nucleus of the hypothalamus (PVN) is a key structure for the regulation of sympathetic outflow and cardiovascular control (11-14), playing thus a major role in bodily homeostasis and neuroendocrine control (15-17). These actions are mediated via direct innervation of sympatheticrelated brainstem and spinal cord neurons $(11-14)$. There is also compelling evidence supporting a critical role for the PVN in the onset and maintenance of sympathohumoral activation during HF (18-22). Importantly, neuroinflammation within the PVN has been described as a hallmark pathophysiological mechanism contributing to increased sympathetic outflow during this disease (23-26). The PVN is also recognized as an important center in emotional regulation and harbors a variety of cells producing different neuropeptides (27-29). In line with this, the PVN directly communicates with the lateral subdivision (CeL) of the central amygdala (CeA), which plays an important role in depression $(30,31)$, fear and anxiety (32-34). Oxytocin-synthesizing neurons in the PVN project long-range axonal terminals to the CeL, where the local release of oxytocin directly regulates anxiety levels $(35,36)$, fear memories and fear extinction $(37,38)$. Moreover, a recent study highlighted the pivotal role of oxytocin signaling in the CeA in mouse models of depression (39). While neuroinflammation in the PVN of HF rats has been previously described $(25,26)$, it is at present unknown whether HF-induced neuroinflammation in other brain areas, such as the CeA also occurs, and if so, how it temporally relates to the onset of neuroinflammation in the PVN.

Neuroinflammation is predominantly mediated by microglia, the immune cells that take up residence in the brain parenchyma $(40,41)$. While classical markers such as increased microglial density, increased expression of ionized calcium-binding adapter molecule 1 (IBA1) and various cytokines are widely used to assess neuroinflammation, they provide only indirect information about detailed microglial morphology changes during this pathological process. This is critical because diverse microglial morphometric features are not only associated to diverse microglial functions $(42,43)$, but more importantly, they have been recently associated to different stages in the spatio-temporal progression of the neuroinflammation process (44). Moreover, there is considerable microglial heterogeneity and brain region-specific differences in size, density or activation stages (45). Therefore, a detailed 3D analysis of microglia cell morphology is needed to overcome these current limitations. Such detailed analysis and quantification of microglial morphometry in the PVN and CeA under control and disease conditions, such as HF has, to the best of our knowledge, not been performed yet, and this constitutes a major goal of the present work. Given that neuroinflammation involves an intricate interplay between microglia and 
astrocytes (46-48), we were also interested to determine whether HF would alter astrocyte function and morphology as well.

To this end, we created a comprehensive glial morphometric profiler and used a novel approach to perform a detailed quantitative analysis of microglial and astrocyte morphology in the PVN and CeA of HF rats in a time-dependent manner 8,14 and 16 weeks (abbreviated as $8 w, 14 w$ and $16 w$ ) after the onset of the disease. Moreover, we correlated these morphological parameters with the assessment of various classical markers for neuroinflammation via qPCR (IBA1, GFAP, TNF-a, IL-1 $\beta$ and IL-6) as well as markers for astrocyte A1 (neurotoxic) and A2 neuroprotective phenotypes (Serping1, C3, Sphk1 and Tm4sf1) (4851). Our results highlight for the first time that in addition to the PVN, HF also induces a robust microglial/astrocyte cell remodeling and activation as well as increased cytokine levels in the CeA, a critical forebrain region involved in emotion and cognition. Neuroinflammation-related changes in the CeA occurred with a delayed time course compared to those in the PVN, and in both cases, correlated with the severity of the disease, suggesting that neuroinflammation could potentially contribute to cognitive impairment and mood disorders observed at later stages of the disease in HF patients.

\section{Materials And Methods}

All performed experiments were approved by the Georgia State University Institutional Animal Care and Use Committee (IACUC) and carried out in agreement with the IACUC guidelines. At all time, animals had ad libitum access to food and water and all efforts were made to minimize suffering and the numbers of animals used for this study.

\section{Animals}

We used male Wistar rats (5-7 weeks old at surgery, 180-200 g, Envigo, Indianapolis, IN, USA) for all experiments (total $n=47$, immunohistochemistry and IMARIS analysis: 29; qPCR: 18). Rats were housed in cages ( 2 per cage) under constant temperature $\left(22 \pm 2{ }^{\circ} \mathrm{C}\right)$ and humidity $(55 \pm 5 \%)$ on a 12 -h light cycle (lights on: 08:00-20:00).

\section{Heart Failure Surgery And Echocardiography}

HF was induced by coronary artery ligation as previously described (21). In brief, animals were anaesthetized using $4 \%$ isoflurane and intubated for mechanical ventilation. To exteriorize the heart, we performed a left thoracotomy. The ligation was performed on the main diagonal branch of the left anterior descending coronary artery. Animals received buprenorphine SR-LAB $(0.5 \mathrm{mg} / \mathrm{kg}$, S.C.; ZooPharm, Windsor, CO, USA) before the surgical procedure to minimize postsurgical pain. Sham animals underwent the same procedure except the occlusion of the left coronary artery. Five weeks after the surgery we performed transthoracic echocardiography (Vevo 3100 systems; Visual Sonics, Toronto, ON; Canada) under light isoflurane (2-3\%) anesthesia. We obtained the left ventricle internal diameter and the left 
diameter of the ventricle posterior and anterior walls in the short-axis motion imaging mode to calculate the ejection fraction (EF). The myocardial infarct surgery typically results in a wide range of functional HF, as determined by the $\mathrm{EF}$ measurements. Rats with $\mathrm{EF}<40 \%$ were considered as fully developed $\mathrm{HF}$ (32.48 $\pm 2.01 \%(n=21)$, whereas those with $E F>50 \%$ were considered to express a mild form of HF $(64.9 \pm 2.1 \%$ $(n=5)$. The average EF in Sham rats was $79.13 \pm 1.57 \%(n=21)$. Unless otherwise indicated (Fig. 6a-d and Fig. d), most studies were compared sham and fully developed HF. Animals were used 8, 14 or 16 weeks after the HF surgery and allocated to the respective groups as indicated in the individual experiments. In addition, we used an additional naive control group, which comprised rats that did not undergo any surgical procedure.

\section{Immunohistochemistry}

Following pentobarbital-induced anesthesia (Euthasol, Virbac, ANADA \#200 - 071, Fort Worth, TX, USA, Pentobarbital, $80 \mathrm{mg} / \mathrm{kgbw}$, i.p.), rats were first perfused at a speed of $20 \mathrm{~mL} / \mathrm{min}$ with $0.01 \mathrm{M} \mathrm{PBS}$ $\left(200 \mathrm{~mL}, 4^{\circ} \mathrm{C}\right.$ ) through the left ventricle followed by $4 \%$ PFA (in $0.3 \mathrm{M} \mathrm{PBS}, 200 \mathrm{~mL}, 4^{\circ} \mathrm{C}$ ), while the right atrium was opened with an incision. Brains were post-fixated for 24 hours in $4 \%$ PFA at $4{ }^{\circ} \mathrm{C}$ and transferred into a $30 \%$ sucrose solution (in $0.01 \mathrm{M} \mathrm{PBS}$ ) at $4{ }^{\circ} \mathrm{C}$ for $3-4$ days. For immunohistochemistry, $40 \mu \mathrm{m}$ slices were cut using a Leica Cryostat (CM3050 S) and brain slices were kept in $0.01 \mathrm{M}$ PBS at $4{ }^{\circ} \mathrm{C}$ until used for staining. Brain slices containing the PVN (A/P, Bregma: $-1.1 \mathrm{~mm}$ to $-1.6 \mathrm{~mm}$ ) and the CeA (A/P, Bregma: $-2.0 \mathrm{~mm}$ to $-2.8 \mathrm{~mm}$ ) were blocked with $5 \%$ Normal Horse Serum in $0.01 \mathrm{M}$ PBS for $1 \mathrm{~h}$ at room temperature. To identify the PVN and CeA topographically, we used the supraoptic nucleus and fornix, as well as the optic tract and commissural stria terminalis as landmarks, respectively. After a 15min washing in $0.01 \mathrm{M}$ PBS, brain slices were incubated for $24 \mathrm{~h}$ in $0.01 \mathrm{M} \mathrm{PBS}, 0.1 \%$ Triton- $\mathrm{X}, 0.04 \% \mathrm{NaN}_{3}$ containing 1:1000 of anti-IBA1 (polyclonal rabbit, Wako, 019-19741, Lot: CAK1997), 1:1000 antiglutamine synthetase (monoclonal mouse, Merck Milipore, MAB 302, clone GS-6) or anti-GFAP (goat polyclonal, abcam, ab53554) at room temperature. Following 15-min washing in 0.01M PBS, sections were incubated in $0.01 \mathrm{M}$ PBS, $0.1 \%$ Triton- $\mathrm{X}, 0.04 \% \mathrm{NaN}_{3}$ with 1:500 Alexa Fluor 488/594-conjugated donkey anti-rabbit/goat/mouse (Jackson ImmunoResearch, 711-585-152, 705-585-147, 715-545-151) for 4 hours at RT. Brain slices were washed again for $15 \mathrm{~min}$ in $0.01 \mathrm{M}$ PBS and mounted using antifade mounting medium (Vectashield $\mathrm{H}-1500$ ).

\section{Confocal Microscopy And 3d Imaris Analysis}

For the 3D reconstruction of microglia, we took Z-stack images (50 $\mu \mathrm{m}$ depth, $1 \mu \mathrm{m}$ steps, 40x magnification) of PVN, CeA (lateral and medial subdivision of the $\mathrm{CeA}$, but not the capsular subdivision) and somatosensory cortex 1 barrel field (S1BF) using a Zeiss LSM 780 confocal microscope (1024 $\times$ 1024 pixel, 16-bit depth, pixel size 0.63 -micron, zoom 0.7). Raw czi files were used for further analysis using IMARIS software (Version 9.31, Oxford Instruments). First, IMARIS was used to reconstruct the microglial surface using the following custom settings: surfaces Detail $0.700 \mu \mathrm{m}$ (smooth); thresholding 
Background subtraction (Local Contrast), diameter of largest Sphere, which fits into the object: 2.00; Color: base, diffusion transparency: $65 \%$. After surface reconstruction, we used the filter function to remove unspecific background signals: Filter: Volume max $-400 \mu \mathrm{m}^{3}$. All microglia with incomplete somata (cut by either the $\mathrm{x}$, $\mathrm{y}$ or $\mathrm{z}$ plane) were manually removed and not included in further analysis. In addition, reconstructed entities that were clearly not microglia (i.e. filaments without soma) were also manually removed. Fused microglia that were falsely recognized as one entity by the software were manually separated using the cut function, or entirely removed from the sample if a separation was not feasible. The 'filter/area function' was used to remove small microglial segments that occurred during manual. After deletion of all background signals the 'mask all' function was used to create the final surface reconstruction. Next, the surface reconstruction was used as the template for the filament reconstruction using the following custom settings: detect new starting points: largest Diameter $7.00 \mu \mathrm{m}$, seed points $0.300 \mu \mathrm{m}$; remove seed points around starting points: diameter of sphere regions: $15 \mu \mathrm{m}$. Seed points were corrected for (either placed in or removed from the center of the somata) manually if the IMARIS algorithm placed them incorrectly. All surface and filament parameters were exported into separate Excel files and used for data analysis. For all quantifications, we used 6-8 40x z-stacks per animal ( 2 z-stacks per brain hemisphere). On average, the reconstruction of a single z-stack took 15 minutes using a computer suited for IMARIS analysis (Intel Core i7 8700 @3.2 GHz, 64 GB RAM, x-64-bit, Windows 10 Enterprise), which included the manual removal of microglia. All images used for analysis were taken with the same confocal settings (pinhole, laser intensity, digital gain and digital offset).

\section{Sholl Analysis}

Sholl analysis was performed using IMARIS in the filament reconstruction mode and individual data sets were exported into separate Excel files for further analysis. We defined a threshold for microglial complexity (peak number for Sholl intersections per microglia): $<10.0$ Intersections (deramified), $>9.9$ (ramified) and we calculated microglial complexity based on these defined thresholds. For the total number of Sholl intersections we added together all the intersections (from each individual sphere) per individual microglia.

\section{Reverse transcription polymerase chain reaction (RT-PCR) and quantitative real time PCR (qPCR)}

RNA extraction and isolation were performed using the miRNAeasy Mini kit (Qiagen, Cat. No. 217004) and the QIAzol Lysis Reagent (Qiagen, Mat. No. 1023537). Briefly, $250 \mu \mathrm{m}$-thick tissue sections were made in cryostat $\left(-20^{\circ} \mathrm{C}\right.$, Leica, CM3050S) and punches from the PVN (2 mm punches, 4 punches per animal), CeA (1 mm punches, 8 punches per animal) and prelimbic cortex (PLC, $2 \mathrm{~mm}$ punches, 4 punches per animal) were collected and kept in dry ice until the RNA extraction procedure. RNA concentration was measured using NanoDrop One (Thermo Scientific) and was in the range of 115$220 \mathrm{ng} / \mu \mathrm{l}$ prior to cDNA synthesis. cDNA synthesis was performed using the iScript ${ }^{\mathrm{TM}}$ gDNA Clear cDNA 
Synthesis Kit (BIO RAD, cat. no. 1725035) and the SimpliAmp Thermal Cycler (applied biosystems, Thermo Fisher Scientific) following the manufacturer's protocol. qPCR was conducted using the following 10x QuantiTect primers (diluted in $1.1 \mathrm{~mL} \mathrm{TE} \mathrm{pH} \mathrm{8.0,} \mathrm{final} \mathrm{concentration:} 200 \mathrm{nM}$ ) purchased from Qiagen: IBA1 (QT01591751), GFAP, IL-1 $\beta$ (QT00181657), IL-6 (QT00182896), Serping1 (QT01607326), C3 (QT00187159), Tm4sf1 (QT01588034), Sphk1 (QT00182035), TNF-a (QT00182896) and $\beta$-Actin (QT00193473, used as the reference gene). All individual qPCR reactions (brain region, primer and condition) were triplicated.

\section{Statistical analysis}

All statistical analyses were performed using GraphPad Prism 8 (GraphPad Software, California, USA). The significance of differences was determined using Student's t test or two-way ANOVA for two-group comparisons followed by Tukey post-hoc test, as indicated throughout the text. For all statistical tests regarding microglial parameters, we calculated the mean value for the respective parameter for each animal and then the mean of the mean (where the final "n" was the number of animals tested) was used for statistical analysis. For the generation and comparison of Sholl distribution curves and to analyze the shift in microglial populations, we used individual microglia values, but still performed the respective statistical tests with the average value of each animal. For qPCR, samples were pooled together in the respective group and we performed a one-sample t-test with technical replicates (triplicates). Results are expressed as mean \pm standard error of the mean (SEM). If not mentioned otherwise, statistical comparisons were performed between HF groups and their respective sham groups. Results were considered statistically significant if $p<0.05$ and are presented as * for $p<0.05$, ** for $p<0.01$ and $* * *$ for $p<0.0001$ in the respective Figures.

\section{Results}

\section{Development and validation of a comprehensive microglia profiler to obtain an in-depth 3D morphometric analysis of various cellular parameters}

To investigate potential HF-induced changes in microglial and astrocyte morphology, as well as proinflammatory cytokines levels and astrocyte markers, we used our established model of HF (Fig. 1a) and performed morphometric and genetic assessments at different time points post-surgery (Fig. 1b). In general, assessment of microglia staining during neuroinflammatory processes has relied on the evaluation of IBA1 immunoreactivity intensity and density within the tissue. However, this approach is insufficient to provide critical information regarding various morphometric microglial parameters such as degree of ramification, somatic volume and overall cellular complexity. Thus, to obtain a detailed quantitative morphometric analyses of glia cells in HF, we used our novel glial profiler based on the IMARIS software to perform 3D reconstruction of microglia and astrocytes. First, we probed for a potential HF-induced microglial proliferation. We did not find significant differences in total microglial cell 
numbers between sham and HF rats in both the PVN (sham 14w: $34.3 \pm 4$ cells vs HF $14 \mathrm{w}: 35.2 \pm 4$ cells, n.s., $n=4$ ) and the CeA (sham 14w: $34.7 \pm 4$ cells vs. HF 14w: $35.1 \pm 3$ cells, n.s., n=4) (Fig. 1c, d), indicative that during HF there is no microglial proliferation within these brain regions at 14 weeks postsurgery.

We then used a more detailed approach to assess changes in individually identified microglia cell surface area, cell volume, filament length, branches and morphometric complexity (Fig. 2a). In brief, we took $50 \mu \mathrm{m}$ z-stack confocal images (16-bit, $1 \mu \mathrm{m}$ steps, $40 \mathrm{x}$ objective) of brain slices stained with IBA1 and exported czi files for further analysis in IMARIS. Incompletely stained microglia were not included into our analysis (detailed description of IMARIS-based analysis can be found in 'Materials and Methods'). This method allows an unbiased high throughput analysis of various cellular features and is a universally applicable tool for morphometric analysis of different cell types under various conditions. To assess whether the sham surgery itself resulted in microglia morphometric alterations over time, we perfused naïve (20 weeks old, $n=2$ ) and sham rats (8-, 14- and 16-weeks post-surgery, n=4/group). We stained brain sections containing the PVN and the CeA with IBA1 and analyzed microglial profiles using IMARIS. We did not observe any significant changes in the PVN or CeA of sham rats in any of these parameters either when compared to naive rats $(20 \mathrm{w})$, or as a function of time after the surgical procedure ( $8 w, 14 w$ or $16 w$, Fig. 3a-d). Therefore, we decided to pool together the three sham groups for further analysis.

\section{HF-induced a time-dependent morphometric microglial changes in the PVN that are already evident at 8 weeks post-surgery}

To study HF-induced microglial changes in the PVN and CeA over time, we perfused sham and HF rats at 8,14 and 16 weeks after the surgery ( $n=4 /$ group) and assessed microglial surface area, cell volume, filament length, microglial branches, microglial segments, filament terminals and IBA1 density.

Representative confocal images of PVN microglia of sham and HF rats 16-weeks post-surgery are shown in Fig. 4a, the anatomical location of the CeA is shown in Fig. 4b. As depicted in Fig. 4b, we found significant morphometric microglial changes already at 8 weeks post-surgery, which progressed as a function of time. We found a progressive decrease in microglial surface area $(0.9 \%, n . s ., 12.3 \%, p=0.0051$ and 17.1\%, $p<0.0001$ at 8, 14, and 16 weeks, respectively, one-way ANOVA, F=11.34, $p<0.0001)$; a progressive decrease in microglial cell volume $(7.0 \%, p=0.0475,27.5 \%, p<0.0001$ and $34.0 \%, p<0.0001$ at 8,14 , and 16 weeks, respectively, one-way ANOVA, F=48.21, $p<0.0001$ ); a decrease in filament length $(10.6 \%, p=0.0002,12.5 \%, p<0.0001$ and $26.1 \%, p<0.0001$ at 8,14 , and 16 weeks, respectively, one-way ANOVA, $F=22.46, p<0.0001)$; a progressive reduction in the number of microglial branches $(17.8 \%$, $p<0.0001,21.0 \%, p<0.0001$ and $26.6 \%, p<0.0001$ at 8,14 , and 16 weeks, respectively, one-way ANOVA, $F=19.33, p<0.0001)$; a progressive reduction in the number of microglial segments $(24.2 \%, p<0.0001$, $26.8 \%, p<0.0001$ and $32.2 \%, p<0.0001$ at 8,14 , and 16 weeks, respectively, one-way ANOVA, F=40.20, 
$p<0.0001)$; a reduction in the number of filament terminals $(22.3 \%, p<0.0001,26.0 \%, p<0.0001$ and $28.1 \%$, $p<0.0001$ at 8,14 , and 16 weeks, respectively, one-way ANOVA, $F=25.9, p<0.0001$ ) and a progressive increase in IBA1 intensity $(17.4 \%, p<0.0001,34.4 \%, p<0.0001$ and $34.6 \%, p<0.0001$ at 8,14 , and 16 weeks, respectively, one-way ANOVA, $F=89.41, p<0.0001$. These findings indicate that HF-induced microglial morphological changes in the PVN are evident as early as 8 weeks after the myocardial infarction.

Next, to rule out that HF-induced changes in microglia morphology was a diffuse phenomenon affecting the whole brain, we chose the primary somatosensory cortex (barrel field, S1BF, Fig. 5a-c) as a functionally-unrelated control region and analyzed IBA1-labeled microglia in this structure. We did not observe any significant microglial changes within this region, suggesting that, at least at this stage cortical brain areas do not display significant changes in microglial morphology.

Lastly, to determine whether the observed microglial changes were dependent on the severity of the disease, we included an additional control group of rats that underwent the myocardial infarction surgery (and used at 16 weeks post-surgery), but that developed only a mild form of functional heart failure, indicated by an $\mathrm{EF}>50 \%$ (mean $\mathrm{EF}: 64.9 \pm 2.1 \%, \mathrm{n}=5$ ). We found that a mild functional HF was not associated to any significant changes of microglial morphology, given that all our tested parameters were similar between our two groups (sham $16 \mathrm{w}$ vs. HF EF>50\% 16w, Fig. 6a-c). Moreover, this additional group allowed us to perform a correlative analysis of the degree of microglia changes as a function of the severity of HF (i.e., as a function of EF value). For this analysis we selected three representative and key microglia morphometric endpoints (cell volume, filament length and number of microglial branches) from 3 experimental groups: sham rats $(n=4,16 w) H F<50 \% \operatorname{EF}(n=4,16 w)$ and $H F>50 \% E F(n=5,16 w)$. As summarized in Figure $6 \mathrm{~d}$, we found a strong and significant correlation for all three morphometric parameters and EF, supporting the notion that morphometric changes in microglia during HF are dependent on the severity of the disease.

\section{HF-induced delayed morphometric microglial changes in the CeA that correlated with the severity of the disease}

Representative confocal images of CeA microglia of sham and HF rats 16-weeks post-surgery are shown in Fig. 7a. In stark contrast to the PVN, except for a slight decrease in filament length, we did not detect any significant changes in microglia morphology 8 weeks after surgery, indicating that HF-induced changes in microglial morphology do not affect the CeA at this point in time. However, some changes in microglial morphometric parameters emerged at 14 weeks, which continued to progress at week 16 . We found a decrease in microglial surface area $(2.4 \%$, n.s. and $15.6 \%, p=0.0063$ at 14 and 16 weeks, respectively, one-way ANOVA, $F=4.821, p=0.034$ ); an initial increase by $21.4 \%, p<0.0001$ followed by a later decrease by $12.7 \%, p=0.028$ in microglial cell volume at 14 and 16 weeks, respectively, one-way ANOVA, $F=12.09, p<0.0001$; a progressive decrease in filament length $(12.0 \%, p=0.014,10.2 \%, p=0.041$ and $28.3 \%, p<0.0001$ at 8,14 and 16 weeks, respectively, one-way ANOVA, $F=10.60, p<0.0001$ ); a progressive reduction in the number of microglial branches $(10.0 \%, p=0.0244$ and $15.1 \%, p=0.012$ at 14 
and 16 weeks, respectively, one-way ANOVA, $F=5.62, p=0.0001$ ); a reduction in the number of microglial segments $(12.1 \%, p=0.01$ and $18.5 \%, p=0.027$ at 14 and 16 weeks, respectively, one-way ANOVA, $F=6.442$, $p<0.0001)$; a progressive reduction in the number of filament terminals $(15.3 \%, p=0.01$ and $21.0 \%$, $p<0.0001$ at 14 and 16 weeks, respectively, one-way ANOVA, $F=9.87, p<0.0001)$ and a progressive increase in IBA1 intensity $(11.1 \%, p<0.0001$ and $25.5 \%, p<0.001$ at 14 and 16 weeks, respectively, one-way ANOVA, $F=51.28, p<0.0001$, Fig. $7 b, c)$. It is important to note that while we observed an initial increase of microglial cell volume at 14 weeks-post surgery in CeA, at 16 weeks post-surgery the cell volume was significantly decreased compared to the sham group. These findings suggest that during HF, microglia undergo cellular/somatic swelling prior to the retraction of processes and decrease in cellular volume.

Similarly to what we observed in the PVN, microglia morphometric parameters were not changes in rats displaying a mild form of $\mathrm{HF}$ (i.e., $\mathrm{EF}>50 \% ; n=5$, see results summarized in Table 1 ). Importantly, we also found key CeA microglia morphometric endpoints (cell volume, filament length and microglial branches) to strongly correlate with the severity of the disease (Fig. 7d). Taken together, these results indicate that CeA microglia undergo morphometric changes during $\mathrm{HF}$, which occurred with a delayed time course compared to PVN microglia and which also correlated to the severity of the disease. The complete overview of all individual values from all groups can be found in Table 1.

\section{HF results in microglial deramification and somatic swelling in both PVN and CeA}

As stated above, pro-inflammatory microglia have been demonstrated to undergo deramification, a process where microglia retract their processes, lose microglial complexity and release inflammatory cytokines $(42,52)$. Thus, to further investigate microglial cell morphometric changes during HF in the PVN and CeA, we performed a Sholl analysis of individually 3D-reconstructed microglial cells in each experimental group. To this end, we superimposed spheres of increasing radius $(1 \mu \mathrm{m}$ increase in radius per step, Fig. 8a) starting at the center of the soma, and measured the number of process intersections that each sphere encountered. We found that in the PVN (Fig. 8b) microglia displayed a significant loss of complexity (indicated by a significantly reduced average number of total Sholl intersections) that occurred in a time-dependent manner with changes being already detectable 8 weeks post-surgery, and becoming progressively more deramified at 14 - and 16-weeks post-surgery (Fig. 8b, $11.1 \%, p=0.008$, $17.1 \%, p<0.0001$ and $30.8 \%, p<0.0001$ at 8,14 , and 16 weeks, respectively, one-way ANOVA, $F=36.3$, p 0.0001 ). In the CeA (Fig. 8c), we did not observe such changes at 8- or 14-weeks post-surgery. However, 16-weeks post-surgery, microglia in the CeA displayed significant deramification (sham: $641.3 \pm 34 \mathrm{vs}$. HF $16 w$ : $534.7 \pm 25, p=0.036$, one-way ANOVA, $F=7.42, p<0.0001$ ). A recent two-dimensional high throughput study on microglia suggested that detailed morphometric analyses of microglia might be more informative and yielded valuable information leading the authors to coin the terms 'low and high-activity' microglia (53). However, given that microglia are highly active cells both at ramified and deramified cellular states, we chose a more descriptive/neutral classification into ramified and deramified (proinflammatory) microglia. To quantify the degree of overall microglia deramification, we first determined 
the peak number of Sholl intersections (i.e., the highest numeric value of a sphere intersecting with a microglial process) per individual microglial cell, which in our entire sampled microglia cell population ranged from 0 to 64 (the higher the number, the more ramified the microglial structure). Using a semiarbitrary and conservative threshold of $<10$ to categorize a microglial cell as pro-inflammatory, we found $32.5 \%$ deramified microglia in the PVN (Fig. 8d) and 30.9\% in the CeA (Fig.8e) of sham rats 14 weeks post-surgery In the HF rats, we found a significant increase in the number of deramified microglia 8 weeks post-surgery in the PVN (HF 14w: $41.4 \%$ vs. sham: $32.5 \%, p=0.0125$, one-way ANOVA, $F=25.70, p<0.0001$ ), but not the $\mathrm{CeA}(31.8 \%$, n.s.). However, 14 weeks post-surgery we found a significant increase in the number of deramified microglia in the CeA $(38.2 \%, p=0.0201$, one-way ANOVA, $F=12.12, p<0.0001)$, while the number of deramified microglia in the PVN had further increased $(55.9 \%, p=0.001)$. In the CeA, the percentage of deramified microglia had further increased 16 weeks post-surgery (HF 16w: $44.0 \%$, $p=0.0163)$, which was not the case for the PVN $(60.25 \%$, n.s. $)$.

Finally, we analyzed the Sholl distribution curves for sham and HF in the PVN and the CeA at 16-weeks post-surgery and also compared microglia between PVN and CeA in sham animals (Fig. 8f-h). We found significant differences for both the PVN (two-way ANOVA, group: $F(1,62)=96.48, p<0.0001$, Fig. 8f) and the CeA (two-way ANOVA, group: $F(1,62)=83.77, \mathrm{p}<0.0001$, Fig. $8 \mathrm{~g}$ ). In the $\mathrm{PVN}$ the mean peak number of Sholl intersections was reached at $21 \mu \mathrm{m}$ distance from the soma for both HF and sham animals, and the mean number of intersections at the peak was significantly different between those groups (sham: 18.5 \pm 1.2 intersections vs. HF $16 \mathrm{w}: 10.2 \pm 0.9$ intersections, $p<0.0001)$. In the CeA, this peak was reached at $22 \mu \mathrm{m}$ for the sham group and $21 \mu \mathrm{m}$ for the HF group and was also significantly different between the two groups (sham: $21.9 \pm 1.6$ intersections vs. HF $16 \mathrm{w}$ : $13.9 \pm 1.3$ intersections, $p<0.0001$ ). Interestingly, under control conditions microglial cells in the CeA were significantly more complex than in the PVN (twoway ANOVA, group: $F(1,62)=74.13, p<0.0001$, Fig. 8h), highlighting the morphological heterogeneity among microglia within different brain regions. These findings suggest that microglia in both PVN and CeA undergo morphometric changes and deramification during HF.

A recent study highlighted that in addition to deramification, pro-inflammatory microglia display somatic swelling (53), a process thought to coincide with the release of pro-inflammatory cytokines, especially in neurodegeneration (54). Thus, to investigate whether HF surgery resulted in somatic microglia swelling, we used our 3D profiler to calculate the somatic volume of individual microglial cells. We found that the average microglial soma volume of sham rats 14 weeks post-surgery was $554.3 \pm 21 \mu \mathrm{m}^{3}$ for the PVN and $529.0 \pm 41 \mu \mathrm{m}^{3}$ for the CeA (Fig. 9a-c). We found a time-dependent increase in somatic volume of PVN microglia: $28.2 \%, p=0.0025$ and $43.1 \%, p<0.0001$ at 14 and 16 weeks, respectively, compared to the respective sham group, one-way ANOVA, $F=12.48, p<0.0001$. In the CeA, we found even more timedependent increases in somatic volume: $41.3 \%, p<0.0001$ and $51.2 \%, p<0.0001$ at 14 and 16 weeks, respectively, compared to the respective sham group, one-way ANOVA, $F=20.37, p<0.0001$.

We next sought to investigate whether somatic swelling and microglial deramification were correlated processes or whether they occurred independently in separate microglial subpopulations. Therefore, we correlated soma volume and the total number of Sholl intersections for each microglial cell (Fig. 9d-g). 
We found that both in sham and HF rats, somatic swelling and deramification were highly correlated processes and this held true for both PVN and CeA ( $p<0.0001$ for all cases). Interestingly, we observed apparent decreases in the slope of the best-fit non-linear regression line (Fig. 9d-g, red lines) in HF compared to sham rats, in both brain regions, which might be indicative of the less polarized (i.e., more homogenous) microglial cell population spectrum in HF rats, resulting from the shift towards a proinflammatory microglial stage. It is important to note that although we found CeA microglia to have a smaller average somata than PVN microglia in sham rats, they surpassed PVN microglia in soma volume at 14 weeks post-surgery. This finding suggests that although somatic swelling and deramification are correlated phenomena, somatic swelling might be initiated earlier and could potentially explain the initial increase in total cell volume in CeA microglia at this time (Fig. 7b, cell volume, HF 14w).

\section{HF induces a morphological A1 astrocyte phenotype in both PVN and CeA}

During injury and disease, microglia and astrocytes display intricate interactions that may lead either to neuronal survival or neuronal loss. A recent study showed that activated (pro-inflammatory) microglia induce a neurotoxic $A 1$ subtype of astrocytes, which secrete a currently unknown neurotoxin that results in neuronal cell death (48). A1 astrocytes can be discriminated from the neuroprotective A2 astrocytes not only by the upregulation of genetic markers $(47-49,55)$, but also by distinct morphological changes, which appear to be similar to those of microglia during neuroinflammation (56). While retraction of astrocytic processes, hypertrophy and gliosis has been described for astrocytes under various conditions (57), no study - to the best of our knowledge - comprehensively addressed alterations in astrocyte morphology during chronic neuroinflammation using a three-dimensional reconstruction profiler. Given that neuroinflammation-induced changes in astrocytes seem to generally follow those observed in microglia $(47,48)$, we chose to investigate astrocytic changes at 14 weeks post-surgery.

To investigate whether HF results in the induction of the A1 astrocytic phenotype, we performed 3D IMARIS analysis of astrocytes (Fig. 10a) that were immunohistochemically identified by their expression of both GFAP and glutamine synthetase (GluSyn) (Fig. 10b, e). We specifically chose a combination of these two markers because they allowed us to perform a detailed analysis of astrocytic somata and processes, given that GFAP stains mostly processes, but not the soma, while GluSyn predominantly stains the astrocyte soma, but not processes (see Fig. 10j). In HF rats, we found significant morphological changes in both PVN and CeA astrocytes 14 weeks post-surgery ( $\mathrm{n}=4$ rats/group) that included a decrease in surface area (17.2\%, $\mathrm{p}=0.0062$ and $19.1 \%, \mathrm{p}=0.022$, for $\mathrm{PVN}$ and CeA, respectively), cell volume (24.4\%, $\mathrm{p}=0.001$ and $23.2 \%, \mathrm{p}=0.001$, for PVN and CeA, respectively), filament length $(19.4 \%$, $p=0.0052$ and $24.6 \%, p=0.0033$, for $P V N$ and CeA, respectively) and an increase in soma volume (30.8\%, $p<0.0001$ and $18.8 \%, p=0.026$, for PVN and CeA, respectively, Fig. 10c, f). Furthermore, we found that astrocytes of $\mathrm{HF}$ rats displayed a significant loss of process complexity, as shown by a significant decrease in the total number of Sholl intersections per astrocyte both in the PVN (18.0\%) and the CeA $(27.8 \%, p<0.0001$ in both cases, Fig. $10 d, g)$. In addition, the Sholl distribution analysis revealed 
significant changes in astrocyte complexity 14-weeks post-surgery for both the PVN (two-way ANOVA, group: $F(1,81)=84.32$, $\mathrm{p}<0.0001$, Fig. $10 \mathrm{~h}$ ) and CeA (two-way ANOVA, group: $F(1,62)=70.92, \mathrm{p}<0.0001$, Fig. 10i). Fig. 10j shows an isolated PVN astrocyte of a HF rat and subsequent surface reconstruction via IMARIS.

\section{HF induces expression of genes associated with neuroinflammation and $\mathrm{A} 1$ astrocyte phenotype in both PVN and CeA}

To determine whether the microglial/astrocyte morphometric changes observed in $\mathrm{HF}$ rats also corresponded with a genetic profile associated to neuroinflammation and/or a shift to an A1 astrocyte phenotype, we performed qPCR of mRNA transcripts of various genes classically associated to neuroinflammation, as well as several $A 2$ and $A 1$ astrocyte-related genes. We analyzed mRNA levels of IBA1 and GFAP, cytokines (TNF-a, IL-1b and IL-6), A1 astrocyte markers (Serping1 and C3) and A2 astrocyte markers (Tm4sf1 and Sphk1) in micropunches obtained from the PVN and CeA of sham and $\mathrm{HF}$ rats at the same time points ( $8 \mathrm{w}$ and $14 \mathrm{w}$ ) at which the morphometric studies were performed.

In the PVN, we found significant changes in tested genes already at 8 weeks post-surgery ( $\mathrm{n}=5$ per group, pooled): an increase in IBA1 (1.344-fold, $p=0.0006$ ), cytokines (IL-1b: 3.51-fold, $p=0.0163$; IL-6: 1.522-fold, $p=0.0067$ and TNF-a: 3.117 -fold, $p=0.0016$ ) and $A 1$ astrocyte markers (Serping1: 2.767 -fold, $p=0.017$ and C3: 1.127-fold, $p=0.0323)$ as well as a significant decrease in GFAP $(-1.583-f o l d, p=0.0006)$ and $A 2$ astrocyte markers (Tm4sf1: -1.373 -fold, $p=0.0005$ and Sphk1: -1.17 -fold, $p=0.0005$, Fig. 11a). When we analyzed the genes again 14 weeks post-surgery (Fig. 11b) ( $n=4$ /group, pooled), the qPCR of PVN tissue yielded comparable results: IBA1: 1.683-fold, $p=0.0089$; GFAP: $-1.563, p=0.0006$; IL-1b: 3.533-fold, $p=0.0134$; IL-6: 1.62-fold, $p=0.0045$; TNF-a: 2.433 -fold, $p=0.0344$; Serping1: 4.2-fold, $p=0.0042$; C3: 1.387fold, $p=0.0237$; Tm4sf1: $-1.897, p<0.0001$, Sphk1: $-1.333, p=0.0012$ ).

In the CeA we found only GFAP (-1.249-fold, $p=0.0004)$, IL-1b (2.383-fold, $p=0.0291)$ and TNF-a (2.367fold, $p=0.004$ ) to be significantly altered 8 weeks post-surgery (Fig. 11c). However, 14 weeks post-surgery (Fig. 11d) we found significant differences in all tested genes, which were comparable to those observed in the PVN: IBA1 (1.433-fold, $p=0.0039)$, GFAP (-1.323-fold, $p=0.0002)$, IL-1b (2.893-fold, $p=0.0066)$, IL-6 (1.757-fold, $p=0.0035)$, TNF-a (2.72-fold, $p<0.0001)$, Serping1 (5.033-fold, $p=0.0009), C 3$ (1.243-fold, $p=0.0404)$, Tm4sf1 (-1.738-fold, $p=0.0004)$ and Sphk1 (-1.57-fold, $p=0.0009)$. We did not observe significant changes either at 8 weeks or 14 weeks post-surgery in the prelimbic cortex (PLC, Fig. 11e, f), suggesting that this cortical area might not be affected by HF at this point in time.

\section{Discussion}




\section{Heart failure-induced neuroinflammation and its physiological and behavioral correlates}

Cardiovascular diseases including stroke and HF are the leading cause of deaths worldwide, and affected individuals suffer from severe physiological and psychological impairments (1). While compounds like angiotensin converting enzyme inhibitors, angiotensin receptor blockers, mineralocorticoid receptor antagonists or $\beta$-blockers have been successfully used to treat the physiological symptoms of $\mathrm{HF}$ patients with reduced EF (58), little to no treatment is available for HF-induced mood and anxiety disorders. In fact, recent studies suggest that the classical use of selective serotonin reuptake inhibitors may not be an efficient way to relieve depression symptoms in HF patients $(59,60)$. Importantly, neuroinflammation and oxidative stress in the PVN and the rostro-lateral medulla have been reported in rats with HF and neurogenic hypertension $(25,61,62)$. It is well-established that inflammation and activation of the renin-angiotensin system drastically increases sympathetic drive, thereby creating a vicious circle with debilitating effects on affected individuals (63). Moreover, intracerebroventricular infusions of minocycline, an anti-inflammatory antibiotic that inhibits microglia cell activation, resulted in significant attenuation of mean arterial pressure and cardiac hypertrophy (61). These changes were accompanied by a reduction in the total number of activated (pro-inflammatory) microglia as well as reduced mRNA levels for IL-1 $\beta$, IL-6 and TNF- $\alpha$ (61). Taken together, these previous studies pinpoint neuroinflammation, particularly microglial cell activation within the PVN, as a key underlying pathophysiological mechanism contributing to the sympathohumoral and cardiovascular complications associated with HF (23-26). Given that the PVN is a key regulatory structure involved in the integration of behavioral, cardiovascular and neuroendocrine homeostatic responses (64-66), and is richly interconnected with a plethora of brain regions $(32,67-69)$, including the amygdala, it is reasonable to speculate that neuroinflammation could also contribute to mood and anxiety disorders associated to HF. In fact, PVN-amygdala connectivity is well-established $(32,67-69)$ and the release of various PVNsynthesized neuropeptides within the amygdala has been shown to directly modulate aggression, fear and depression $(37,38,70)$. However, to the best of our knowledge, no previous study demonstrated that $\mathrm{HF}$ is associated with a pro-inflammatory microglial profile consisting of somatic swelling and a shift from a ramified to a deramified state.

\section{Microglial deramification in the paraventricular nucleus and central amygdala in heart failure rats}

Microglia, the resident immune cells of the brain parenchyma are among the first responding cells during injury, cell death or unwanted intruders that might access the brain due to a compromised blood-brainbarrier $(40,42,43,52,71)$. Microglia represent a major component in the immune response and participate in the neuroinflammatory response largely via the release pro-inflammatory cytokines $(42,52)$. During neuroinflammation, microglia undergo a morphological transition from a highly ramified to a deramified state, retracting their fine processes while simultaneously undergoing somatic hypertrophy 
$(42,43,72)$. Although it seems evident that a comprehensive knowledge of microglial deramification is of paramount importance, very little is known about the precise series of events that ultimately lead to deramification, highlighting the need for tools that allow a detailed morphometric analysis of microglial remodeling. While other laboratories employed comparable approaches for the reconstruction of microglia under various conditions using different software (73-75), these approaches are very timeconsuming ( 80 minutes or more per z-stack), and could result in over- and/or undersampling given that fused (microglia recognized as one entity by the software) or cut microglia (somata or processes cut by either the $x, y$ or $z$ axis) are included in the analysis. These limitations are largely overcome by our glial profiler based on the IMARIS software which allows for an unbiased, rapid, and fully automated reconstruction and morphometric analysis of individually identified microglial cell. Moreover, our profiler identifies and either separates or eliminates incomplete or fused microglial profiles. While this is not the first study to implement IMARIS analysis for three-dimensional reconstruction of microglia $(76,77)$, our in-depth analysis of microglia morphology and their morphometric transition to a pro-inflammatory state in the context of the progression and severity of a disease model represents, to the best of our knowledge, an unprecedented approach. Morphometric analysis of microglia is superior to classical IHC-based quantification, as it not only provides information about overall microglia-related immunoreactivity density and intensity, but also provides insights into a variety of specific morphological changes within individually identified microglial cell, such as somatic swelling and process remodeling (53). With our 3D profiler, we were able to detect time-dependent microglia morphological changes in HF rats including filament shortening, deramification and somatic swelling in both PVN and CeA. These changes are widely accepted as indicators of a pro-inflammatory microglial state $(42,43,52,53,73)$. We found that both in PVN and CeA, microglial cell remodeling worsened over time (Figs. 4, 7), which is highlighted in part by a progressive increase in the number of deramified microglia (Fig. 8) and a shift of the microglial population towards less complex microglial phenotype. Although the retraction of microglial processes and somatic swelling were highly correlated (Fig. 9), we found an initial increase in total cell volume in the CeA (14w, Fig. 7) suggesting that somatic swelling might precede the deramification. Another important finding of the current study is that processes make up the majority of microglial volume (5-6x more volume in processes than in somata, see Figs. 4, 7 and 9), providing an explanation for the overall decrease in cell volume despite the observed increase in somatic volume following HF. Our data also suggest that HF-induced microglial cell changes were not representative of a diffused phenomenon globally affecting the brain, given that non-related regions, such as S1BF and the PLC (Fig. 5, 11) were not affected, at least at this time point of the disease. Finally, it is important to note that we found significant differences in cell volume, surface area and IBA1 intensity when we compared microglial cells between the PVN and the CeA in sham rats (Fig. 8). These results are in line with previous studies performed both in rodents and humans supporting a high degree of variability of microglial phenotypes that might depend on age, brain region and pathophysiological condition $(43,78,79)$.

\section{Region-specific Differences And Time-dependency Of Heart Failure-induced Neuroinflammation}

Page 15/39 
We found that microglial cell remodeling in the PVN is present already at $8 \mathrm{w}$ post-surgery, while microglial changes in the CeA started to unfold around the 14-week mark. While our study demonstrated for the first time HF-induced microglial cell remodeling in the $\mathrm{CeA}$, a major finding was that these changes occurred in a delayed manner compared to the PVN. These time-dependent microglial structural changes were consistent, and correlated as well with qPCR analysis of pro-inflammatory markers, such as TNF- $a$, IL-1 $\beta$ and IL-6, which in the PVN were already significantly elevated at $8 \mathrm{w}$, while in the CeA most markers became significantly elevated at $14 \mathrm{w}$ post-surgery. These results further support the previously established relationship between microglial cell morphological changes with the release of proinflammatory cytokines $(42,43)$.

As stated above, a large body of evidence supports a key role for the PVN in the onset and maintenance of cardiovascular dysregulation in HF $(11,18-22)$. Thus, the time-dependent differences in microglial deramification and pro-inflammatory markers between the PVN and the amygdala are in line with the fact that sympathohumoral and cardiovascular manifestations in HF precede the mood and cognitive deficits associated with this disease $(58,80,81)$. HF-induced cognitive impairments in both rats and mice has been previously described $(82,83)$ and while it is well established that the PVN is a main neuronal substrate contributing to mood regulation, our studies provide an indirect evidence that neuroinflammation within the amygdala may constitute a neuronal substrate contributing to HF-induced mood deficits. A recent study showed that the microglia inhibitor minocycline improved depression-like behavior in HF rats (84). However, the authors performed oral delivery of minocycline so that the precise site of action within the brain underlying this improvement could not to be determined. Thus, further studies are clearly necessary to demonstrate that neuroinflammation within the CeA contributes to HFinduced depression-like behavioral changes. The mechanisms underlying the time course differences between the HF-induced neuroinflammation in PVN and CeA could be manifold and not mutually exclusive. Based on the tightly coupled interaction between the PVN and the amygdala (32, 67-69), neuroinflammation in the amygdala could be triggered by the exaggerated neuronal activity in the PVN reported in $\operatorname{HF}(21,22,85,86)$. This is in line with previous studies showing that aberrant neuronal activity could result in the release of damage-associated molecules, leading to microglia activation $(87,88)$. Recent studies also support the ability of inflammatory cytokines to diffuse in the extracellular space (89-91). Thus, the delay for neuroinflammation onset in the amygdala could be due to the slow lateral diffusion of cytokines initially originating in the PVN. Finally, the delay may also be due to differential responsiveness of PVN and amygdala microglial cell populations to the same noxious stimulus (i.e. HFassociated hypoxia) and/or differences in blood-brain-barrier integrity, between the PVN and CeA during disease states, which could result in region-specific permeability differences, as we recently showed to be the case during hypertension (92). Lastly, it is important to highlight that we found a strong correlation between key microglia morphometric parameters (both in PVN and CeA) and the degree of functional heart failure, as measured by \%EF, suggesting that microglia morphometric changes were also dependent on the severity of the disease. These findings are in line with human studies supporting that the progression of both cognitive decline (93) and depression (94) might directly be linked to the ejection fraction / severity of the disease. 
Clearly, future studies are warranted to address the precise mechanisms underlying the differential and region-specific time course of pro-inflammatory microglial states during HF.

\section{Morphological changes in PVN and CeA astrocytes and shift towards A1 phenotype}

The intricate interaction between microglia and astrocytes during neuroinflammation is well-established $(56,95,96)$. Microglial cells release various pro-inflammatory molecules upon injury $(97)$ or as a result of the general immune response (98), which in turn triggers specific astrocytic responses. Astrocytes can release orosomucoid-2 to inhibit pro-inflammatory microglia, block the chemokine receptor type 5 of the microglial membrane, downregulate the inflammatory response or further promote neuroinflammation and neurodegeneration (96). This bidirectional interaction allows an efficient and tailored response to various potentially harmful threats that the brain might be exposed to. Recent studies suggested the classification of astrocytes into two categories: A1 (pro-inflammatory, neurotoxic) and A2 (antiinflammatory, neuroprotective) astrocytes (47-49). During the transition from the A2 to A1 phenotype, astrocytes are thought to undergo morphometric changes comparable to those observed in microglia. Based on this, we applied a 3D morphometric analysis in analogy to our microglial analysis to the study of astrocytes in the PVN and CeA of HF rats. Similar to what we found with microglial cells, our results demonstrated significant morphological changes in both PVN and CeA astrocytes during HF. Importantly, these morphometric changes were accompanied by the upregulation of A1-specific (Serping1, C3) and downregulation of A2-specific (Tm4sf1 and Sphk1) astrocyte markers. Taken together, these results indicate that HF promotes a shift in astrocyte phenotype from A2 to A1 both in the PVN and CeA, contributing thus along microglial cells to the overall neuroinflammatory state in this disease. It is important to note that while we analyzed changes in astrocyte morphology only at 14 weeks post-surgery, the time differences in the A1/A2 mRNA markers also support a time-dependent difference between PVN and $\mathrm{CeA}$ in astrocyte changes that coincides with the time course of microglia changes. Interestingly, we found a significantly decreased GFAP mRNA levels in HF rats of both PVN and CeA. While these findings might seem contrary to the general notion that GFAP protein levels are increased in A1 astrocytes, recent studies suggest that GFAP might not be the best marker for astrocyte reactivity (47) and highlight that the relationship between reactive astrocytes and GFAP levels might have been oversimplified (99). Lastly, it is important to note that while we report here significant changes in glial morphology and cytokine levels, it remains unclear whether these changes translate into altered glial function and how they might ultimately affect the pro-inflammatory state within the respective brain regions. Finally, most published studies in the field using the congestive HF rodent model, including the present one, were performed in young adult rats (6-10 weeks), $(23,26,100-102)$. This is a caveat that needs to be taken into consideration, given that the incidence of HF in humans is higher in the elderly population.

Taken together, our findings suggest that HF-induced neuroinflammation not only alters the microglial environment in affected brain regions, but rather has far-reaching consequences affecting astrocytes and potentially even neurons due to astrocyte-induced neurotoxicity $(47,48)$. Additional studies assessing 
neuronal apoptosis are needed to determine whether the HF-induced A1 astrocyte phenotype in the CeA becomes neurotoxic.

\section{Conclusion}

In summary, in the present study we implemented a novel and improved quantitative morphometric imaging approach to assess precise microglial and astrocyte structural remodeling in different brain regions during the progression of heart failure. We provide evidence that in addition to the previously described neuroinflammation observed in the hypothalamic PVN, known to be implicated in sympathohumoral activation during this disease, microglia and astrocytes within the central amygdala (CeA) also undergo structural remodeling. These structural changes are indicative of glial shift towards a pro-inflammatory microglial and a potentially neurotoxic astrocyte phenotype, a finding supported also by an increased expression of pro-inflammatory cytokines and A1/2 astrocyte markers. Importantly, our results show that neuroinflammation in the CeA manifested with a delayed time course with respect to the PVN and were dependent on the severity of the disease. Taken together, our studies support the idea that neuroinflammation in the amygdala may contribute to emotional and cognitive deficits commonly observed at later stages during the course of HF, standing thus as a potential novel target for alleviating comorbid mood disorders in this prevalent disease $(2,4,103)$.

\section{Abbreviations}

C3 - Complement component 3, CeA - Central amygdala, CeL - Lateral subdivision of the central amygdala, , GFAP - Glial fibrillary acidic protein, GluSyn - Glutamine Synthetase, HF - heart failure, IL Interleukin, PLC - Prelimbic cortex, Serping1 - C1-inhibitor gene, Sphk1 - Sphingosine kinase 1, Tm4sf1 - Transmembrane 4 L6 family member 1, TNF-a - Tumor necrosis factor a.

\section{Declarations}

\section{Acknowledgements}

We thank Atit Patel and Dr. Daniel Cox (Georgia State University) for their assistance with the qPCR. We also thank Dr. Anne Murphy (Georgia State University) for kindly providing the GFAP antibody.

\section{Authors' contributions}

FA performed immunohistochemistry, IMARIS reconstruction, analyzed the data and drafted the manuscript; FA and HCFN performed qPCR; HCFN and RKR performed the coronary artery ligation surgery and echocardiographic assessment of EF; MR developed the IMARIS microglial profiler; JES supervised the experiments, designed the study and edited the manuscript. All authors critically reviewed the manuscript and approved the final version for publication.

\section{Funding}


This work was supported by DFG Postdoc Fellowship AL 2466/1-1 to FA; National Heart, Lung, and Blood Institute Grant NIH HL090948, and funding provided by the Center for Neuroinflammation and Cardiometabolic Diseases (CNCD) at Georgia State University.

\section{Availability of data and materials}

The datasets used and/or analyzed in the current study are available from the corresponding author upon reasonable request.

\section{Ethics approval and consent to participate}

All experiments were performed following the approval of the Ethics Committee for Animal Experiments at Georgia State University (IACUC protocol \# A18003). All efforts were made to minimize the suffering of the animals and reduce their total number.

\section{Consent for publication}

Not applicable.

\section{Competing interests}

The author declare that they have no competing interests.

\section{References}

1. Group WCRCW. World Health Organization cardiovascular disease risk charts: revised models to estimate risk in 21 global regions. Lancet Glob Health. 2019;7(10):e1332-e45.

2. Mbakwem A, Aina F, Amadi C. Expert Opinion-Depression in Patients with Heart Failure: Is Enough Being Done? Card Fail Rev. 2016;2(2):110-2.

3. Parissis JT, Fountoulaki K, Paraskevaidis I, Kremastinos D. Depression in chronic heart failure: novel pathophysiological mechanisms and therapeutic approaches. Expert Opin Investig Drugs. 2005;14(5):567-77.

4. Rustad JK, Stern TA, Hebert KA, Musselman DL. Diagnosis and treatment of depression in patients with congestive heart failure: a review of the literature. Prim Care Companion CNS Disord. 2013;15(4).

5. Konstam V, Moser DK, De Jong MJ. Depression and anxiety in heart failure. J Card Fail. 2005;11(6):455-63.

6. Rutledge T, Reis VA, Linke SE, Greenberg BH, Mills PJ. Depression in heart failure a meta-analytic review of prevalence, intervention effects, and associations with clinical outcomes. J Am Coll Cardiol. 2006;48(8):1527-37.

7. Hammond CA, Blades NJ, Chaudhry SI, Dodson JA, Longstreth WT, Jr., Heckbert SR, et al. Long-Term Cognitive Decline After Newly Diagnosed Heart Failure: Longitudinal Analysis in the CHS 
(Cardiovascular Health Study). Circ Heart Fail. 2018;11(3):e004476.

8. Frey A, Popp S, Post A, Langer S, Lehmann M, Hofmann U, et al. Experimental heart failure causes depression-like behavior together with differential regulation of inflammatory and structural genes in the brain. Front Behav Neurosci. 2014;8:376.

9. Prickaerts J, Raaijmakers W, Blokland A. Effects of myocardial infarction and captopril therapy on anxiety-related behaviors in the rat. Physiol Behav. 1996;60(1):43-50.

10. Schoemaker RG, Smits JF. Behavioral changes following chronic myocardial infarction in rats. Physiol Behav. 1994;56(3):585-9.

11. Benarroch EE. Paraventricular nucleus, stress response, and cardiovascular disease. Clin Auton Res. 2005;15(4):254-63.

12. Coote JH, Yang Z, Pyner S, Deering J. Control of sympathetic outflows by the hypothalamic paraventricular nucleus. Clin Exp Pharmacol Physiol. 1998;25(6):461-3.

13. Kannan $\mathrm{H}$, Yamashita $\mathrm{H}$. Electrophysiological study of paraventricular nucleus neurons projecting to the dorsomedial medulla and their response to baroreceptor stimulation in rats. Brain Res. 1983;279(1-2):31-40.

14. Ranson RN, Motawei K, Pyner S, Coote JH. The paraventricular nucleus of the hypothalamus sends efferents to the spinal cord of the rat that closely appose sympathetic preganglionic neurones projecting to the stellate ganglion. Exp Brain Res. 1998;120(2):164-72.

15. Althammer F, Grinevich V. Diversity of oxytocin neurons: beyond magno- and parvocellular cell types? J Neuroendocrinol. 2017.

16. Stern JE. Neuroendocrine-autonomic integration in the paraventricular nucleus: novel roles for dendritically released neuropeptides. J Neuroendocrinol. 2015;27(6):487-97.

17. Atasoy D, Betley JN, Su HH, Sternson SM. Deconstruction of a neural circuit for hunger. Nature. 2012;488(7410):172-7.

18. Patel KP. Role of paraventricular nucleus in mediating sympathetic outflow in heart failure. Heart Fail Rev. 2000;5(1):73-86.

19. Pyner S. The paraventricular nucleus and heart failure. Exp Physiol. 2014;99(2):332-9.

20. Ramchandra R, Hood SG, Frithiof R, McKinley MJ, May CN. The role of the paraventricular nucleus of the hypothalamus in the regulation of cardiac and renal sympathetic nerve activity in conscious normal and heart failure sheep. J Physiol. 2013;591(1):93-107.

21. Biancardi VC, Son SJ, Sonner PM, Zheng H, Patel KP, Stern JE. Contribution of central nervous system endothelial nitric oxide synthase to neurohumoral activation in heart failure rats. Hypertension. 2011;58(3):454-63.

22. Potapenko ES, Biancardi VC, Florschutz RM, Ryu PD, Stern JE. Inhibitory-excitatory synaptic balance is shifted toward increased excitation in magnocellular neurosecretory cells of heart failure rats. $\mathrm{J}$ Neurophysiol. 2011;106(3):1545-57. 
23. Najjar F, Ahmad M, Lagace D, Leenen FHH. Sex differences in depression-like behavior and neuroinflammation in rats post-MI: role of estrogens. Am J Physiol Heart Circ Physiol. 2018;315(5):H1159-H73.

24. Najjar F, Ahmad M, Lagace D, Leenen FHH. Role of Myocardial Infarction-Induced Neuroinflammation for Depression-Like Behavior and Heart Failure in Ovariectomized Female Rats. Neuroscience. 2019;415:201-14.

25. Kang YM, Ma Y, Elks C, Zheng JP, Yang ZM, Francis J. Cross-talk between cytokines and reninangiotensin in hypothalamic paraventricular nucleus in heart failure: role of nuclear factor-kappaB. Cardiovasc Res. 2008;79(4):671-8.

26. Yu Y, Wei SG, Weiss RM, Felder RB. Angiotensin II Type 1a Receptors in the Subfornical Organ Modulate Neuroinflammation in the Hypothalamic Paraventricular Nucleus in Heart Failure Rats. Neuroscience. 2018;381:46-58.

27. Wotjak CT, Kubota M, Liebsch G, Montkowski A, Holsboer F, Neumann I, et al. Release of vasopressin within the rat paraventricular nucleus in response to emotional stress: a novel mechanism of regulating adrenocorticotropic hormone secretion? J Neurosci. 1996;16(23):7725-32.

28. Herman JP, Tasker JG. Paraventricular Hypothalamic Mechanisms of Chronic Stress Adaptation. Front Endocrinol (Lausanne). 2016;7:137.

29. Myers B, McKlveen JM, Herman JP. Glucocorticoid actions on synapses, circuits, and behavior: implications for the energetics of stress. Front Neuroendocrinol. 2014;35(2):180-96.

30. Townsend JD, Eberhart NK, Bookheimer SY, Eisenberger NI, Foland-Ross LC, Cook IA, et al. fMRI activation in the amygdala and the orbitofrontal cortex in unmedicated subjects with major depressive disorder. Psychiatry Res. 2010;183(3):209-17.

31. Young KD, Siegle GJ, Zotev V, Phillips R, Misaki M, Yuan H, et al. Randomized Clinical Trial of RealTime fMRI Amygdala Neurofeedback for Major Depressive Disorder: Effects on Symptoms and Autobiographical Memory Recall. Am J Psychiatry. 2017;174(8):748-55.

32. LeDoux J. The amygdala. Curr Biol. 2007;17(20):R868-74.

33. LeDoux J. The emotional brain, fear, and the amygdala. Cell Mol Neurobiol. 2003;23(4-5):727-38.

34. LeDoux JE. Emotion circuits in the brain. Annu Rev Neurosci. 2000;23:155-84.

35. Sobota R, Mihara T, Forrest A, Featherstone RE, Siegel SJ. Oxytocin reduces amygdala activity, increases social interactions, and reduces anxiety-like behavior irrespective of NMDAR antagonism. Behav Neurosci. 2015;129(4):389-98.

36. Labuschagne I, Phan KL, Wood A, Angstadt M, Chua P, Heinrichs M, et al. Oxytocin attenuates amygdala reactivity to fear in generalized social anxiety disorder. Neuropsychopharmacology. 2010;35(12):2403-13.

37. Hasan MT, Althammer F, Silva da Gouveia M, Goyon S, Eliava M, Lefevre A, et al. A Fear Memory Engram and Its Plasticity in the Hypothalamic Oxytocin System. Neuron. 2019;103(1):133-46 e8. 
38. Knobloch HS, Charlet A, Hoffmann LC, Eliava M, Khrulev S, Cetin AH, et al. Evoked axonal oxytocin release in the central amygdala attenuates fear response. Neuron. 2012;73(3):553-66.

39. Han RT, Kim YB, Park EH, Kim JY, Ryu C, Kim HY, et al. Long-Term Isolation Elicits Depression and Anxiety-Related Behaviors by Reducing Oxytocin-Induced GABAergic Transmission in Central Amygdala. Front Mol Neurosci. 2018;11:246.

40. Nimmerjahn A, Kirchhoff F, Helmchen F. Resting microglial cells are highly dynamic surveillants of brain parenchyma in vivo. Science. 2005;308(5726):1314-8.

41. Raivich G. Like cops on the beat: the active role of resting microglia. Trends Neurosci. 2005;28(11):571-3.

42. Prinz M, Jung S, Priller J. Microglia Biology: One Century of Evolving Concepts. Cell. 2019;179(2):292-311.

43. Prinz M, Priller J. Microglia and brain macrophages in the molecular age: from origin to neuropsychiatric disease. Nat Rev Neurosci. 2014;15(5):300-12.

44. Morrison HW, Filosa JA. A quantitative spatiotemporal analysis of microglia morphology during ischemic stroke and reperfusion. J Neuroinflammation. 2013;10:4.

45. Stratoulias V, Venero JL, Tremblay ME, Joseph B. Microglial subtypes: diversity within the microglial community. EMBO J. 2019;38(17):e101997.

46. Colombo E, Farina C. Astrocytes: Key Regulators of Neuroinflammation. Trends Immunol. 2016;37(9):608-20.

47. Liddelow SA, Barres BA. Reactive Astrocytes: Production, Function, and Therapeutic Potential. Immunity. 2017;46(6):957-67.

48. Liddelow SA, Guttenplan KA, Clarke LE, Bennett FC, Bohlen CJ, Schirmer L, et al. Neurotoxic reactive astrocytes are induced by activated microglia. Nature. 2017;541(7638):481-7.

49. Clarke LE, Liddelow SA, Chakraborty C, Munch AE, Heiman M, Barres BA. Normal aging induces A1like astrocyte reactivity. Proc Natl Acad Sci U S A. 2018;115(8):E1896-E905.

50. Tassoni A, Farkhondeh V, Itoh Y, Itoh N, Sofroniew MV, Voskuhl RR. The astrocyte transcriptome in EAE optic neuritis shows complement activation and reveals a sex difference in astrocytic C3 expression. Sci Rep. 2019;9(1):10010.

51. Zamanian JL, Xu L, Foo LC, Nouri N, Zhou L, Giffard RG, et al. Genomic analysis of reactive astrogliosis. J Neurosci. 2012;32(18):6391-410.

52. Butovsky 0 , Weiner HL. Microglial signatures and their role in health and disease. Nat Rev Neurosci. 2018;19(10):622-35.

53. Davis BM, Salinas-Navarro M, Cordeiro MF, Moons L, De Groef L. Characterizing microglia activation: a spatial statistics approach to maximize information extraction. Sci Rep. 2017;7(1):1576.

54. Hickman S, Izzy S, Sen P, Morsett L, El Khoury J. Microglia in neurodegeneration. Nat Neurosci. 2018;21(10):1359-69. 
55. Neal M, Luo J, Harischandra DS, Gordon R, Sarkar S, Jin H, et al. Prokineticin-2 promotes chemotaxis and alternative A2 reactivity of astrocytes. Glia. 2018;66(10):2137-57.

56. Burda JE, Sofroniew MV. Seducing astrocytes to the dark side. Cell Res. 2017;27(6):726-7.

57. Li T, Chen X, Zhang C, Zhang Y, Yao W. An update on reactive astrocytes in chronic pain. J Neuroinflammation. 2019;16(1):140.

58. Metra M, Teerlink JR. Heart failure. Lancet. 2017;390(10106):1981-95.

59. Rajeswaran T, Plymen CM, Doherty AM. The effect of antidepressant medications in the management of heart failure on outcomes: mortality, cardiovascular function and depression - a systematic review. Int J Psychiatry Clin Pract. 2018;22(3):164-9.

60. Ricco J, Benson J, Prasad S. PURLs: SSRIs for depression/heart failure patients? Not so fast. J Fam Pract. 2017;66(9):564-7.

61. Shi P, Diez-Freire C, Jun JY, Qi Y, Katovich MJ, Li Q, et al. Brain microglial cytokines in neurogenic hypertension. Hypertension. 2010;56(2):297-303.

62. Wu KL, Chan SH, Chan JY. Neuroinflammation and oxidative stress in rostral ventrolateral medulla contribute to neurogenic hypertension induced by systemic inflammation. J Neuroinflammation. 2012;9:212.

63. Diaz HS, Toledo C, Andrade DC, Marcus NJ, Rio RD. Neuroinflammation in heart failure: NEW insights for an old disease. J Physiol. 2019.

64. Swanson LW, Kuypers HG. The paraventricular nucleus of the hypothalamus: cytoarchitectonic subdivisions and organization of projections to the pituitary, dorsal vagal complex, and spinal cord as demonstrated by retrograde fluorescence double-labeling methods. J Comp Neurol. 1980;194(3):555-70.

65. Swanson LW, Sawchenko PE. Paraventricular nucleus: a site for the integration of neuroendocrine and autonomic mechanisms. Neuroendocrinology. 1980;31(6):410-7.

66. Guyenet PG. The sympathetic control of blood pressure. Nat Rev Neurosci. 2006;7(5):335-46.

67. Kim TK, Han PL. Functional Connectivity of Basolateral Amygdala Neurons Carrying Orexin Receptors and Melanin-concentrating Hormone Receptors in Regulating Sociability and Mood-related Behaviors. Exp Neurobiol. 2016;25(6):307-17.

68. Pittman QJ, Blume HW, Renaud LP. Connections of the hypothalamic paraventricular nucleus with the neurohypophysis, median eminence, amygdala, lateral septum and midbrain periaqueductal gray: an electrophysiological study in the rat. Brain Res. 1981;215(1-2):15-28.

69. Huber D, Veinante P, Stoop R. Vasopressin and oxytocin excite distinct neuronal populations in the central amygdala. Science. 2005;308(5719):245-8.

70. Bosch OJ, Neumann ID. Both oxytocin and vasopressin are mediators of maternal care and aggression in rodents: from central release to sites of action. Horm Behav. 2012;61(3):293-303.

71. Thurgur H, Pinteaux E. Microglia in the Neurovascular Unit: Blood-Brain Barrier-microglia Interactions After Central Nervous System Disorders. Neuroscience. 2019;405:55-67. 
72. Lannes N, Eppler E, Etemad S, Yotovski P, Filgueira L. Microglia at center stage: a comprehensive review about the versatile and unique residential macrophages of the central nervous system. Oncotarget. 2017;8(69):114393-413.

73. Heindl S, Gesierich B, Benakis C, Llovera G, Duering M, Liesz A. Automated Morphological Analysis of Microglia After Stroke. Front Cell Neurosci. 2018;12:106.

74. Xu H, Gelyana E, Rajsombath M, Yang T, Li S, Selkoe D. Environmental Enrichment Potently Prevents Microglia-Mediated Neuroinflammation by Human Amyloid beta-Protein Oligomers. J Neurosci. 2016;36(35):9041-56.

75. Karperien A, Ahammer H, Jelinek HF. Quantitating the subtleties of microglial morphology with fractal analysis. Front Cell Neurosci. 2013;7:3.

76. Erny D, Hrabe de Angelis AL, Jaitin D, Wieghofer P, Staszewski O, David E, et al. Host microbiota constantly control maturation and function of microglia in the CNS. Nat Neurosci. 2015;18(7):96577.

77. VanRyzin JW, Marquardt AE, Argue KJ, Vecchiarelli HA, Ashton SE, Arambula SE, et al. Microglial Phagocytosis of Newborn Cells Is Induced by Endocannabinoids and Sculpts Sex Differences in Juvenile Rat Social Play. Neuron. 2019;102(2):435-49 e6.

78. Dubbelaar ML, Kracht L, Eggen BJL, Boddeke E. The Kaleidoscope of Microglial Phenotypes. Front Immunol. 2018;9:1753.

79. Bottcher C, Schlickeiser S, Sneeboer MAM, Kunkel D, Knop A, Paza E, et al. Human microglia regional heterogeneity and phenotypes determined by multiplexed single-cell mass cytometry. Nat Neurosci. 2019;22(1):78-90.

80. Leto L, Feola M. Cognitive impairment in heart failure patients. J Geriatr Cardiol. 2014;11(4):316-28.

81. Celutkiene J, Vaitkevicius A, Jakstiene S, Jatuzis D. Expert Opinion-Cognitive Decline in Heart Failure: More Attention is Needed. Card Fail Rev. 2016;2(2):106-9.

82. Toledo C, Lucero C, Andrade DC, Diaz HS, Schwarz KG, Pereyra KV, et al. Cognitive impairment in heart failure is associated with altered Wnt signaling in the hippocampus. Aging (Albany NY). 2019;11(16):5924-42.

83. Hay M, Vanderah TW, Samareh-Jahani F, Constantopoulos E, Uprety AR, Barnes CA, et al. Cognitive impairment in heart failure: A protective role for angiotensin-(1-7). Behav Neurosci. 2017;131(1):99114.

84. Wang HW, Ahmad M, Jadayel R, Najjar F, Lagace D, Leenen FHH. Inhibition of inflammation by minocycline improves heart failure and depression-like behaviour in rats after myocardial infarction. PLoS One. 2019;14(6):e0217437.

85. Roy RK, Augustine RA, Brown CH, Schwenke DO. Activation of oxytocin neurons in the paraventricular nucleus drives cardiac sympathetic nerve activation following myocardial infarction in rats. Commun Biol. 2018;1:160.

86. Zheng H, Li YF, Wang W, Patel KP. Enhanced angiotensin-mediated excitation of renal sympathetic nerve activity within the paraventricular nucleus of anesthetized rats with heart failure. Am J Physiol 
Regul Integr Comp Physiol. 2009;297(5):R1364-74.

87. Wohleb ES. Neuron-Microglia Interactions in Mental Health Disorders: "For Better, and For Worse". Front Immunol. 2016;7:544.

88. Nair A, Bonneau RH. Stress-induced elevation of glucocorticoids increases microglia proliferation through NMDA receptor activation. J Neuroimmunol. 2006;171(1-2):72-85.

89. Tchelingerian JL, Quinonero J, Booss J, Jacque C. Localization of TNF alpha and IL-1 alpha immunoreactivities in striatal neurons after surgical injury to the hippocampus. Neuron. 1993;10(2):213-24.

90. Vitkovic L, Konsman JP, Bockaert J, Dantzer R, Homburger V, Jacque C. Cytokine signals propagate through the brain. Mol Psychiatry. 2000;5(6):604-15.

91. Stojkovic T, Colin C, Le Saux F, Jacque C. Specific pattern of nitric oxide synthase expression in glial cells after hippocampal injury. Glia. 1998;22(4):329-37.

92. Biancardi VC, Stern JE. Compromised blood-brain barrier permeability: novel mechanism by which circulating angiotensin II signals to sympathoexcitatory centres during hypertension. J Physiol. 2016;594(6):1591-600.

93. Zuccala G, Cattel C, Manes-Gravina E, Di Niro MG, Cocchi A, Bernabei R. Left ventricular dysfunction: a clue to cognitive impairment in older patients with heart failure. J Neurol Neurosurg Psychiatry. 1997;63(4):509-12.

94. Jiang W, Alexander J, Christopher E, Kuchibhatla M, Gaulden LH, Cuffe MS, et al. Relationship of depression to increased risk of mortality and rehospitalization in patients with congestive heart failure. Arch Intern Med. 2001;161(15):1849-56.

95. Sofroniew MV. Astrocyte barriers to neurotoxic inflammation. Nat Rev Neurosci. 2015;16(5):249-63.

96. Jha MK, Jo M, Kim JH, Suk K. Microglia-Astrocyte Crosstalk: An Intimate Molecular Conversation. Neuroscientist. 2019;25(3):227-40.

97. Bellver-Landete V, Bretheau F, Mailhot B, Vallieres N, Lessard M, Janelle ME, et al. Microglia are an essential component of the neuroprotective scar that forms after spinal cord injury. Nat Commun. 2019;10(1):518.

98. Rivest S. Regulation of innate immune responses in the brain. Nat Rev Immunol. 2009;9(6):429-39.

99. Adams KL, Gallo V. The diversity and disparity of the glial scar. Nat Neurosci. 2018;21(1):9-15.

100. Zheng H, Katsurada K, Liu X, Knuepfer MM, Patel KP. Specific Afferent Renal Denervation Prevents Reduction in Neuronal Nitric Oxide Synthase Within the Paraventricular Nucleus in Rats With Chronic Heart Failure. Hypertension. 2018;72(3):667-75.

101. Kang YM, Gao F, Li HH, Cardinale JP, Elks C, Zang WJ, et al. NF-kappaB in the paraventricular nucleus modulates neurotransmitters and contributes to sympathoexcitation in heart failure. Basic Res Cardiol. 2011;106(6):1087-97.

102. Potapenko ES, Biancardi VC, Zhou Y, Stern JE. Altered astrocyte glutamate transporter regulation of hypothalamic neurosecretory neurons in heart failure rats. Am J Physiol Regul Integr Comp Physiol. 
2012;303(3):R291-300.

103. Celano CM, Daunis DJ, Lokko HN, Campbell KA, Huffman JC. Anxiety Disorders and Cardiovascular Disease. Curr Psychiatry Rep. 2016;18(11):101.

\section{Tables}




\begin{tabular}{|c|c|c|c|c|c|c|c|c|}
\hline Brain region & Group & $\begin{array}{c}\text { Cell } \\
\text { volume } \\
\left(\mu \mathrm{m}^{3}\right)\end{array}$ & $\begin{array}{c}\text { Surface } \\
\text { area } \\
\left(\mu \mathrm{m}^{2}\right)\end{array}$ & $\begin{array}{l}\text { Filament } \\
\text { length } \\
(\mu \mathrm{m})\end{array}$ & $\begin{array}{l}\text { Dendritic } \\
\text { branches }\end{array}$ & $\begin{array}{l}\text { Dendritic } \\
\text { segments }\end{array}$ & $\begin{array}{l}\text { Filament } \\
\text { terminals }\end{array}$ & $\begin{array}{c}\text { IBA1 } \\
\text { intensity } \\
(\mathrm{AU}) \\
\end{array}$ \\
\hline \multirow{7}{*}{ PVN } & $\begin{array}{c}\text { Sham } 8 w \\
N=381 \\
n=4\end{array}$ & $3157 \pm 112$ & $4048 \pm 99$ & $745 \pm 21$ & $104 \pm 3$ & $219 \pm 7$ & $116 \pm 4$ & $9449 \pm 123$ \\
\hline & $\begin{array}{c}\text { Sham } 14 \mathrm{w} \\
\mathrm{N}=365 \\
\mathrm{n}=4\end{array}$ & $3098 \pm 119$ & $4100 \pm 107$ & $736 \pm 18$ & $102 \pm 4$ & $224 \pm 8$ & $118 \pm 6$ & $9385 \pm 135$ \\
\hline & $\begin{array}{c}\text { Sham 16w } \\
\begin{array}{c}\mathrm{N}=389 \\
\mathrm{n}=4\end{array}\end{array}$ & $3181 \pm 122$ & $4068 \pm 81$ & $761 \pm 25$ & $100 \pm 5$ & $216 \pm 7$ & $117 \pm 6$ & $9522 \pm 139$ \\
\hline & $\begin{array}{c}\text { HF 8w } \\
\mathrm{N}=378 \\
\mathrm{n}=4\end{array}$ & $2838 \pm 58$ & $3868 \pm 75$ & $642 \pm 12$ & $82 \pm 2$ & $171 \pm 5$ & $90 \pm 3$ & $11138 \pm 118$ \\
\hline & $\begin{array}{c}\mathrm{HF} 14 \mathrm{w} \\
\mathrm{N}=407 \\
\mathrm{n}=4\end{array}$ & $2212 \pm 74$ & $3424 \pm 64$ & $629 \pm 35$ & $78 \pm 2$ & $163 \pm 7$ & $85 \pm 3$ & $12764 \pm 202$ \\
\hline & $\begin{array}{c}\mathrm{HF} 16 \mathrm{w} \\
\mathrm{N}=399 \\
\mathrm{n}=4\end{array}$ & $2012 \pm 69$ & $3233 \pm 59$ & $524 \pm 39$ & $72 \pm 3$ & $150 \pm 5$ & $83 \pm 4$ & $12783 \pm 231$ \\
\hline & $\begin{array}{c}\text { HF 16w EF }>50 \% \\
\begin{array}{c}\mathrm{N}=435 \\
n=5\end{array}\end{array}$ & $3234 \pm 128$ & $3988 \pm 90$ & $741 \pm 27$ & $97 \pm 6$ & $216 \pm 8$ & $115 \pm 9$ & $9433 \pm 146$ \\
\hline \multirow{7}{*}{$\mathrm{CeA}$} & $\begin{array}{c}\text { Sham 8w } \\
\mathrm{N}=377 \\
\mathrm{n}=4\end{array}$ & $3588 \pm 137$ & $4885 \pm 119$ & $841 \pm 28$ & $109 \pm 6$ & $219 \pm 7$ & $114 \pm 3$ & $10134 \pm 137$ \\
\hline & $\begin{array}{c}\text { Sham } 14 w \\
\mathrm{~N}=339 \\
\mathrm{n}=4\end{array}$ & $3644 \pm 122$ & $4966 \pm 131$ & $865 \pm 31$ & $102 \pm 2$ & $214 \pm 5$ & $116 \pm 4$ & $10073 \pm 144$ \\
\hline & $\begin{array}{c}\text { Sham 16w } \\
\mathrm{N}=354 \\
\mathrm{n}=4\end{array}$ & $3691 \pm 123$ & $4904 \pm 127$ & $833 \pm 24$ & $104 \pm 5$ & $216 \pm 7$ & $114 \pm 3$ & $10285 \pm 180$ \\
\hline & $\begin{array}{c}\mathrm{HF} 8 \mathrm{w} \\
\mathrm{N}=363 \\
\mathrm{n}=4\end{array}$ & $3634 \pm 119$ & $4700 \pm 124$ & $765 \pm 29$ & $106 \pm 4$ & $222 \pm 7$ & $112 \pm 3$ & $10344 \pm 176$ \\
\hline & $\begin{array}{c}\mathrm{HF} 14 \mathrm{w} \\
\mathrm{N}=355 \\
\mathrm{n}=4\end{array}$ & $4418 \pm 187$ & $4964 \pm 144$ & $781 \pm 28$ & $92 \pm 4$ & $192 \pm 8$ & $100 \pm 3$ & $11314 \pm 277$ \\
\hline & $\begin{array}{c}\mathrm{HF} 16 \mathrm{w} \\
\mathrm{N}=332 \\
\mathrm{n}=4\end{array}$ & $3180 \pm 115$ & $4395 \pm 163$ & $623 \pm 24$ & $89 \pm 3$ & $179 \pm 6$ & $94 \pm 4$ & $12779 \pm 357$ \\
\hline & $\begin{array}{c}\text { HF 16w EF }>50 \% \\
\mathrm{~N}=384 \\
\mathrm{n}=5\end{array}$ & $3540 \pm 132$ & $4798 \pm 141$ & $835 \pm 56$ & $94 \pm 4$ & $202 \pm 7$ & $110 \pm 4$ & $11322 \pm 290$ \\
\hline \multirow[t]{2}{*}{$\mathrm{S} 1 \mathrm{BF}$} & $\begin{array}{c}\text { Sham } 14 \mathrm{w} \\
\mathrm{N}=139 \\
\mathrm{n}=4\end{array}$ & $4577 \pm 138$ & $6008 \pm 234$ & $967 \pm 31$ & $125 \pm 5$ & $261 \pm 8$ & $135 \pm 7$ & $10539 \pm 183$ \\
\hline & $\begin{array}{c}\mathrm{HF} 14 \mathrm{w} \\
\mathrm{N}=162 \\
\mathrm{n}=4\end{array}$ & $4257 \pm 130$ & $5664 \pm 224$ & $957 \pm 31$ & $126 \pm 4$ & $264 \pm 8$ & $138 \pm 8$ & $10647 \pm 171$ \\
\hline
\end{tabular}

Table 1: Summary of microglial morphometric parameters of sham and HF rats for the PVN and CeA Table shows individual values (mean \pm SEM) of all analyzed microglial parameters, $\mathrm{N}=$ number of analyzed microglia, 
$\mathrm{n}=$ number of animals per group, $\mathrm{CeA}=$ central amygdala, $\mathrm{PVN}=$ paraventricular nucleus, $\mathrm{S} 1 \mathrm{BF}=$ somatosensory cortex barrel field 1 .

\section{Figures}

(A)

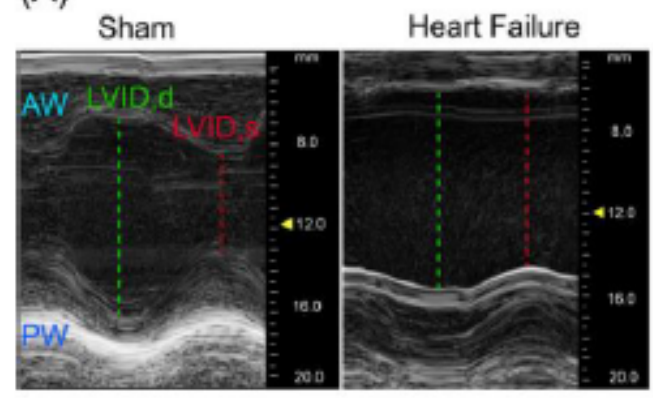

(C)

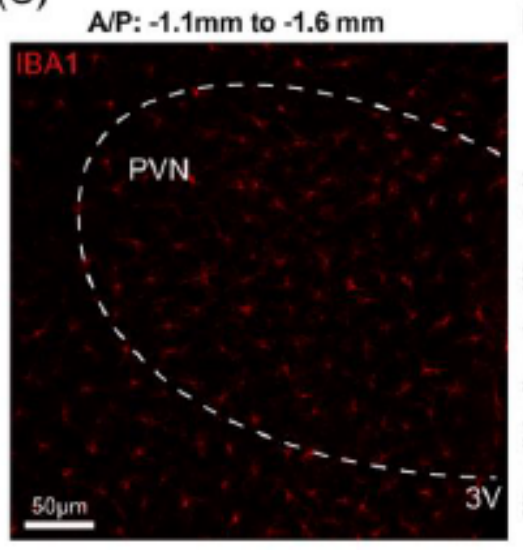

PVN microglia count

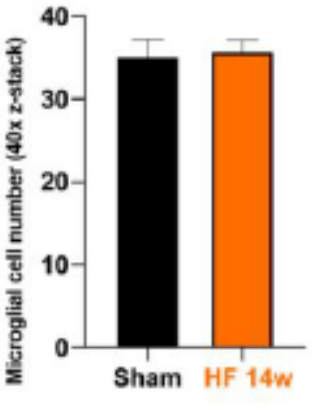

(B)

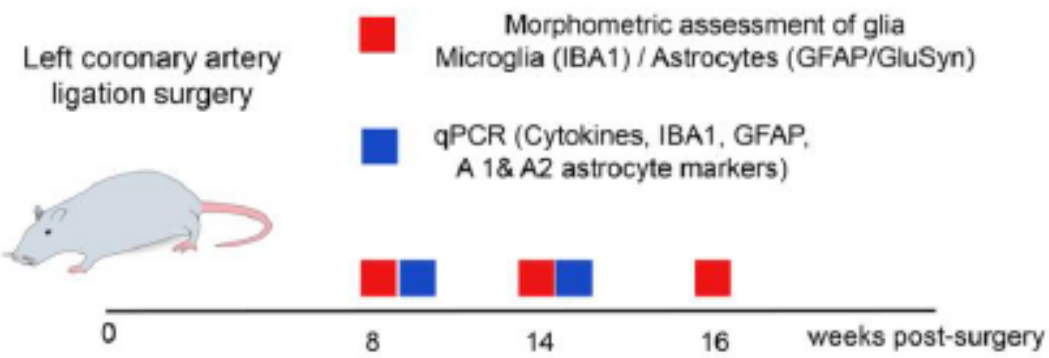

(D)

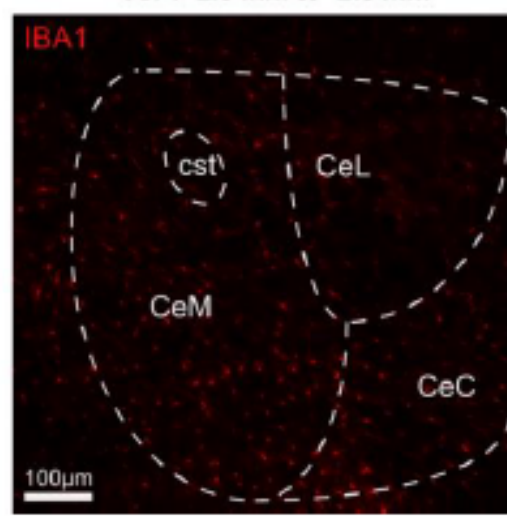

CeA microglia count

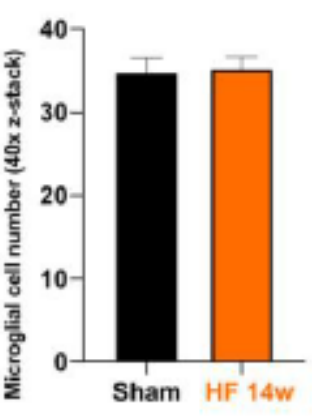

\section{Figure 1}

Heart failure does not alter microglial cell density in the PVN or CeA (a) Representative image showing the echocardiographic assessment left ventricular cardiac function in a sham and HF rats. (b) timetable depicting morphometric assessments and qPCR at 8-, 14- and 16- weeks post-surgery, (c, d) Representative confocal images showing IBA1-labeled microglia (red) in the PVN and CeA. Bar graphs show quantification of microglial cell numbers within the PVN and CeA in sham and HF rats 14 weeks post-surgery, one-way ANOVA was used to analyze the data. 3V: third ventricle, AW: anterior wall, CeC: central amygdala capsular division, CeL: central amygdala lateral division, CeM: central amygdala medial division, cst: commissural stria terminalis, LVID, $d$ and LVID, s: left ventricle internal dimensions in diastole and systole, PVN: paraventricular nucleus, PW: posterior wall. 


\section{(A)}

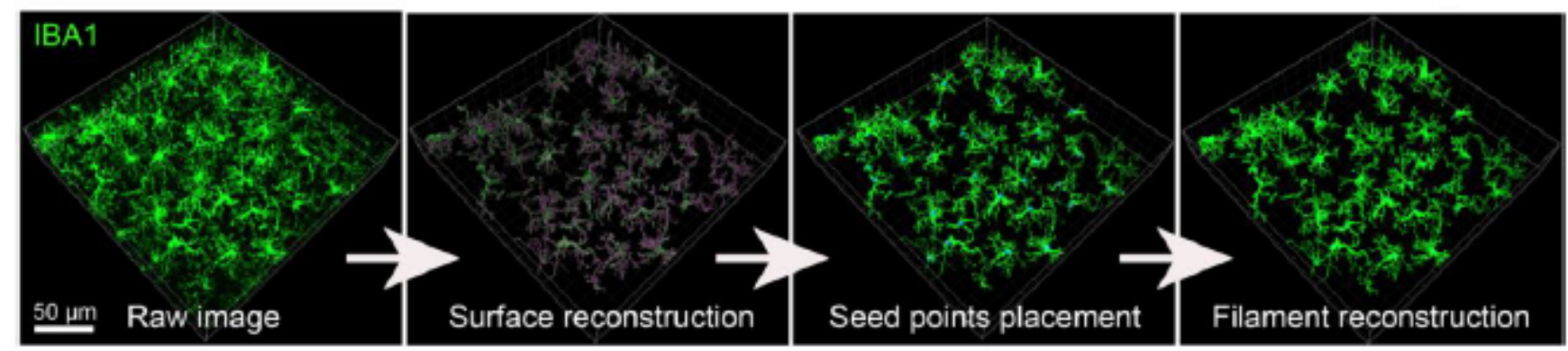

Analysis

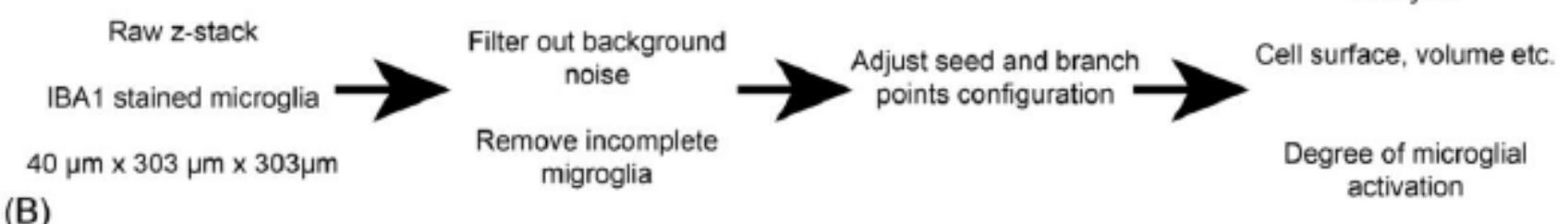

(B)

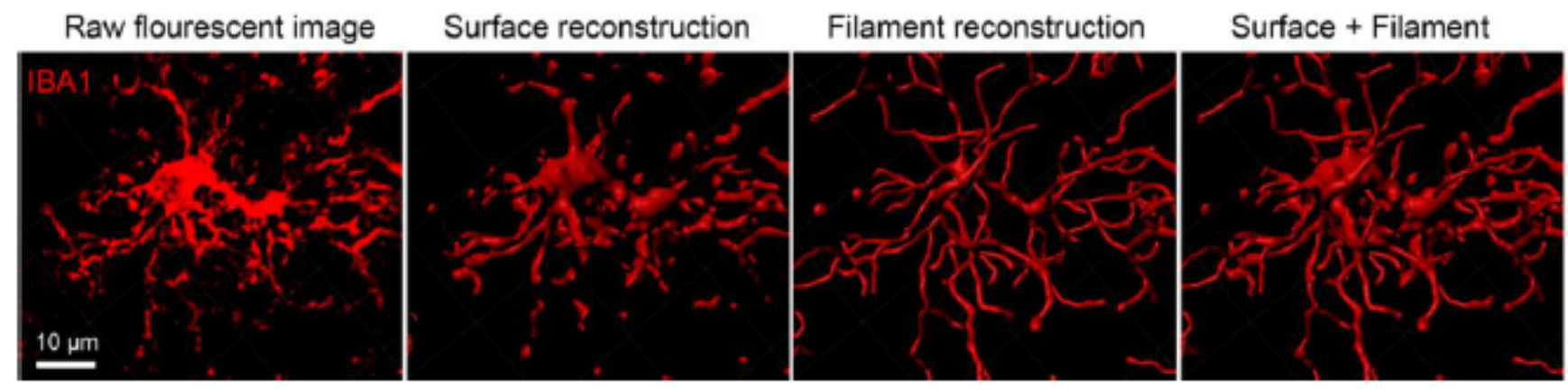

Figure 2

Three-dimensional reconstruction of microglia surface and filaments using IMARIS (a) schematic depiction of the sequential steps used for the 3D morphometric reconstruction and assessment of microglia (IBA1, green), (b) confocal images show the step-by-step process for three-dimensional reconstruction of a single microglia (IBA1, red). For image processing, colors were changed from red to green in IMARIS for better visibility and increased precision during manual editing of reconstructed microglia. Note the merging of red (raw fluorescence) and green (surface reconstruction) channels in the top panel. 
(A)

Sham surgery - no left coronary artery ligation
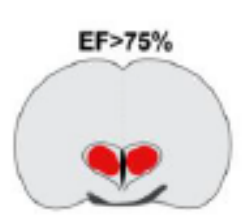

PVN

A.P: $-1.1 \mathrm{~mm}$ to $-1.6 \mathrm{~mm}$

(C)

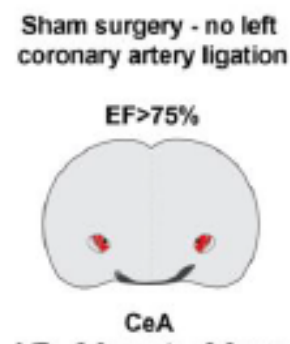

A.P: $-2.0 \mathrm{~mm}$ to $-2.8 \mathrm{~mm}$

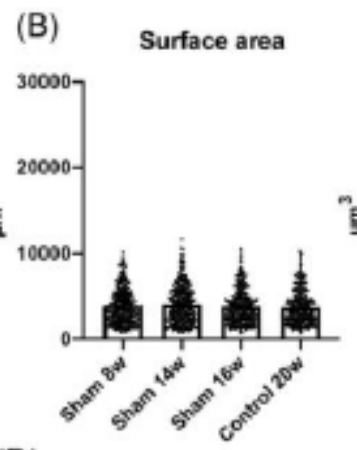

(D)

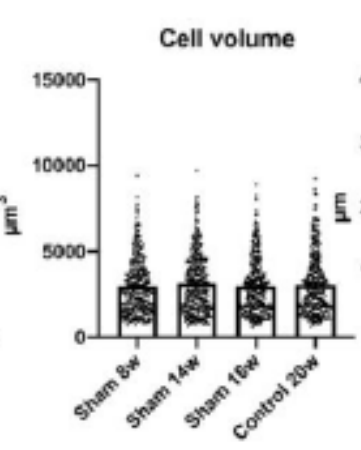

Cell volume

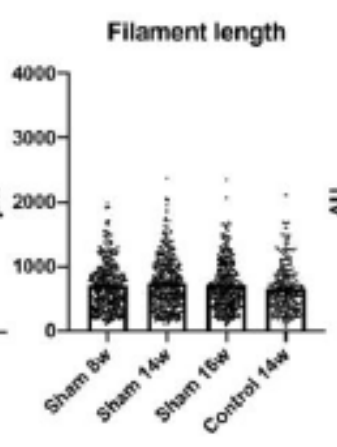

Filament length

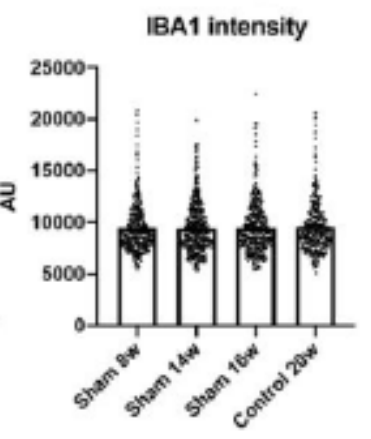

IBA1 intensity
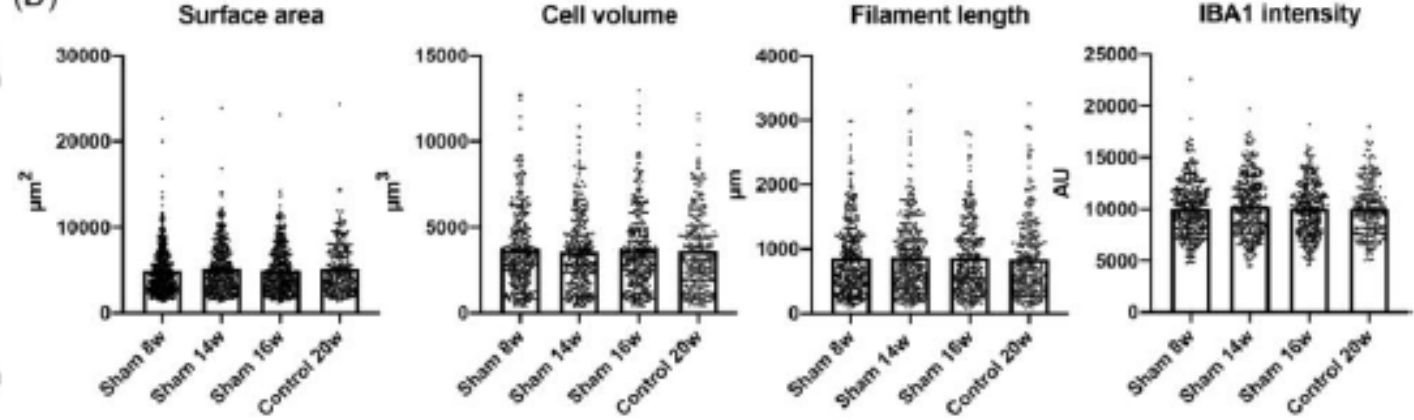

\section{Figure 3}

Sham surgery per se does not alter the morphology of PVN or CeA microglia (a) brain scheme shows the topographic location of PVN microglia that have been used for the morphometric assessment. The red area within the PVN (heart-shaped nucleus) highlights the fraction of the nucleus where pictures were taken, (b) dot-plot graphs show the individual values of PVN microglia for surface area, cell volume, filament length and IBA 1 intensity for the non-surgery control ( $N=357$ from 4 rats), and sham rats at 8,14 and 16 weeks post-surgery ( $N=381$ from 4 rats, $N=365$ from 4 rats and $n=389$ from 4 rats, respectively), one-way ANOVA was used to analyze the data followed by Tukey post-hoc test, (c) brain scheme shows the topographic location of CeA microglia that have been used for the morphometric assessment. The red area within the CeA highlights the divisions where pictures were taken, (d) dot-plot graphs show the individual values of CeA microglia for surface area, cell volume, filament length and IBA 1 intensity for the non-surgery control ( $\mathrm{N}=297$ from 4 rats), and sham rats at 8, 14 and 16 weeks post-surgery ( $\mathrm{N}=377$ from 4 rats, $\mathrm{N}=339$ from 4 rats and $\mathrm{N}=354$ from 4 rats, respectively). 
(A)

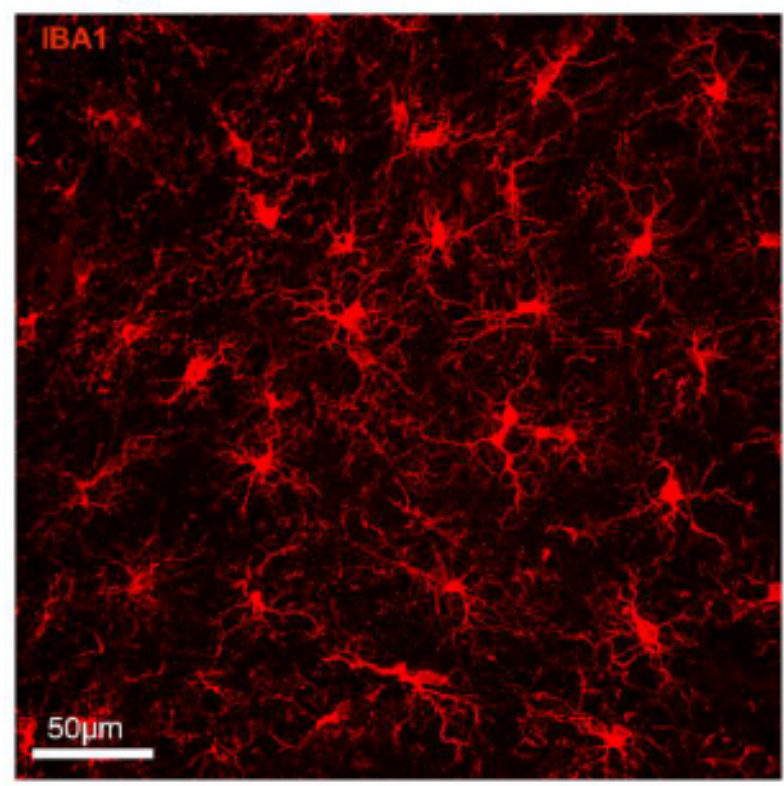

(B)

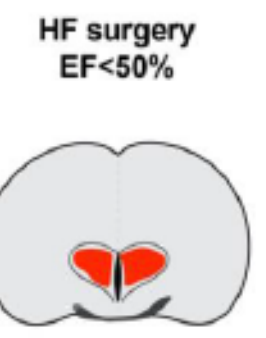

PVN

A/P: $-1.1 \mathrm{~mm}$ to $-1.6 \mathrm{~mm}$

Microglial branches

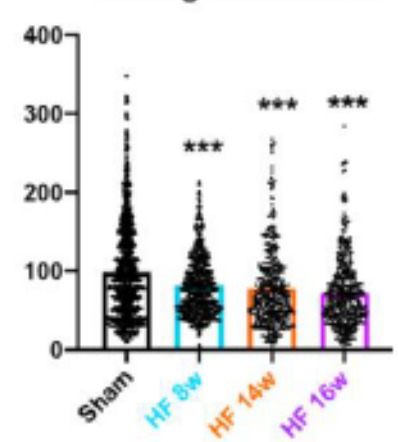

(C)

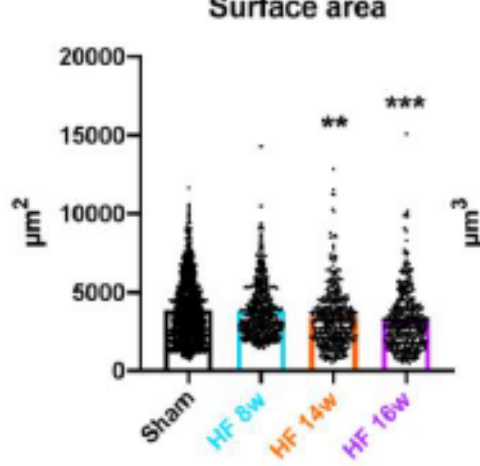

Microglial segments

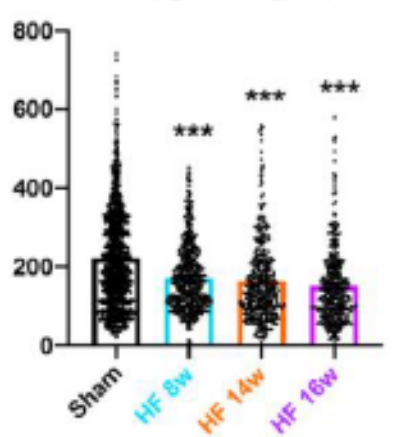

PVN microglia HF $16 \mathrm{w}$

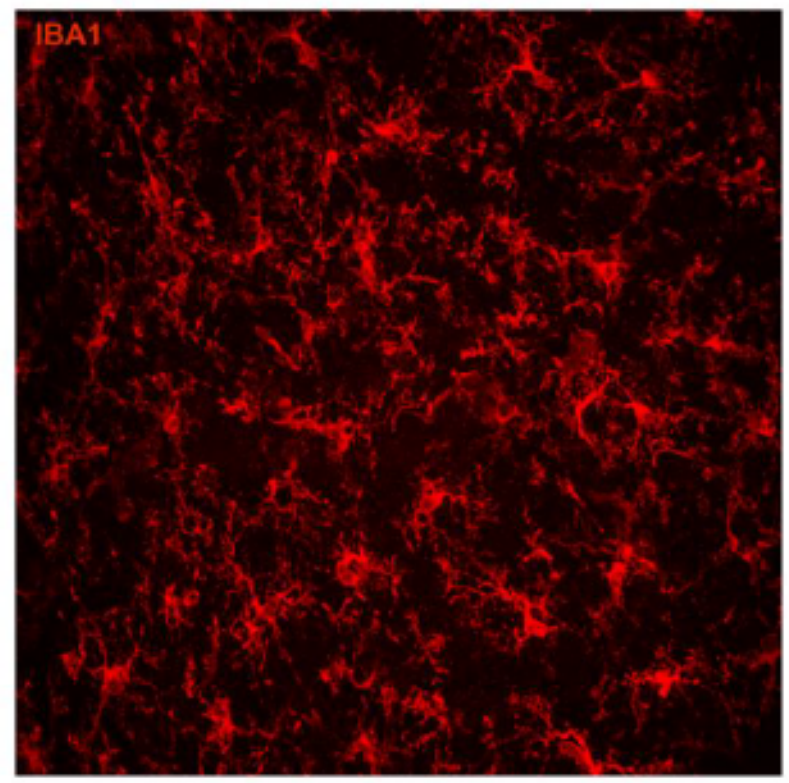

Cell volume

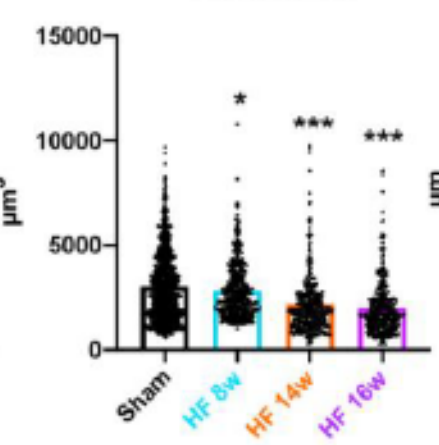

Filament terminals

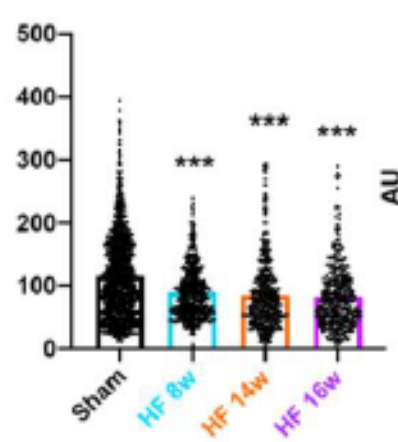

Filament length

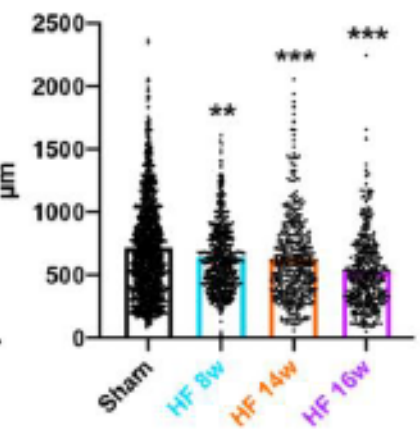

IBA1 intensity

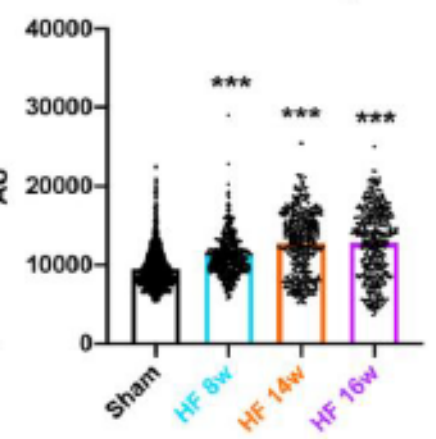

Figure 4

HF-induced morphometric changes in PVN microglia (a) representative confocal images show IBA1stained microglia in the PVN of HF and sham rats 16 weeks post-surgery, (b) brain scheme shows the topographic location of PVN microglia that have been used for the morphometric assessment. The red area within the PVN (heart-shaped nucleus) highlights the fraction of the nucleus where pictures were taken , (c) dot-plot graphs show the individual values of PVN microglia for surface area, cell volume, 
filament length, microglial branches, microglial segments, filament terminals and IBA 1 intensity for sham rats $(\mathrm{N}=1135$ from 12 rats, pooled) and $\mathrm{HF}$ rats at 8,14 and 16 weeks post-surgery ( $\mathrm{N}=378$ from 4 rats, $\mathrm{N}=407$ from 4 rats and $\mathrm{N}=399$ from 4 rats, respectively). ${ }^{*} \mathrm{p}<0.05,{ }^{* \star} \mathrm{p}<0.01$ and ${ }^{* \star *} \mathrm{p}<0.0001$ vs. respective sham, one-way ANOVA followed by Tukey post-hoc test.

(A) S1BF microglia sham 16w

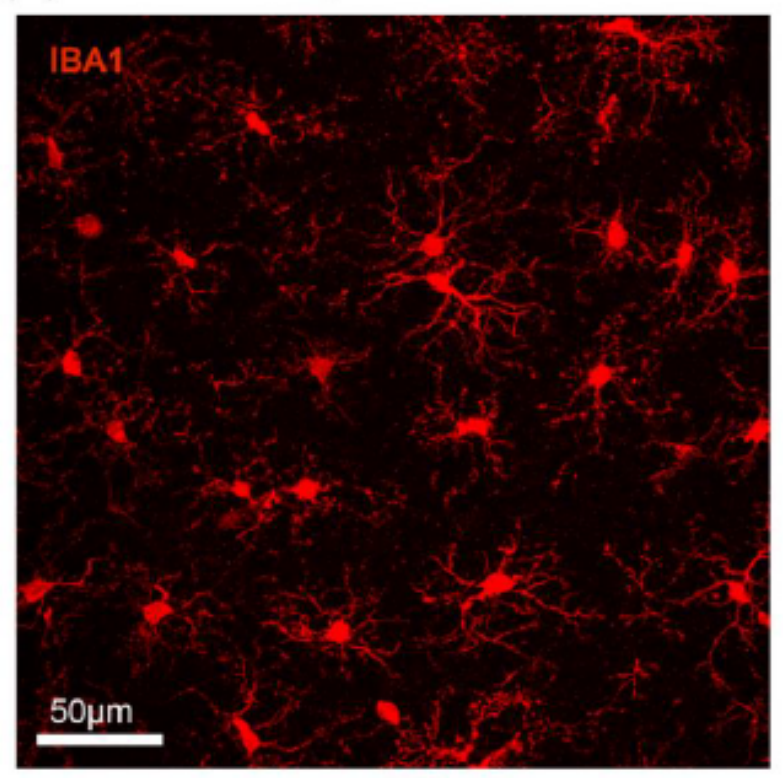

(B)

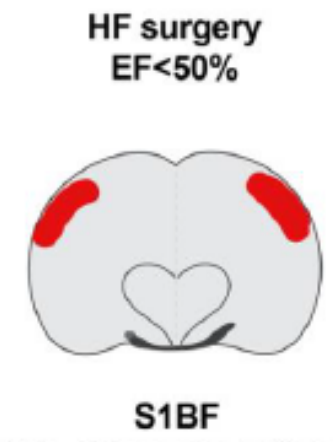

$A / P:-1.1 m m$ to $-2.3 \mathrm{~mm}$
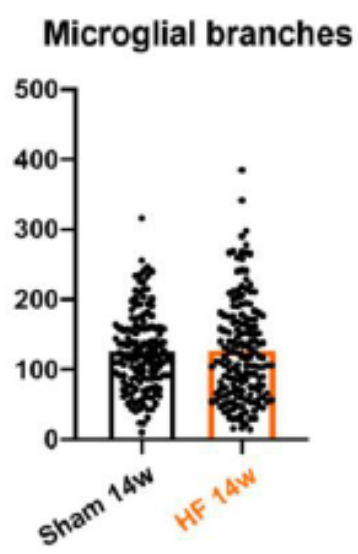

(C)

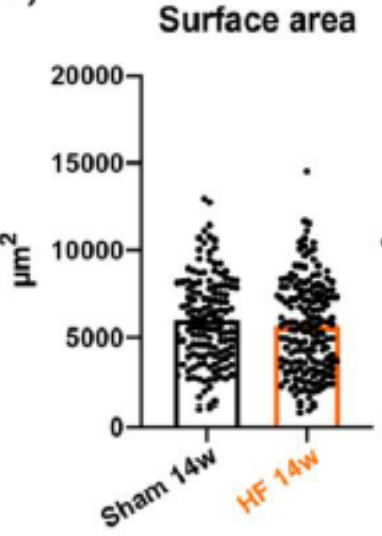

Microglial segments

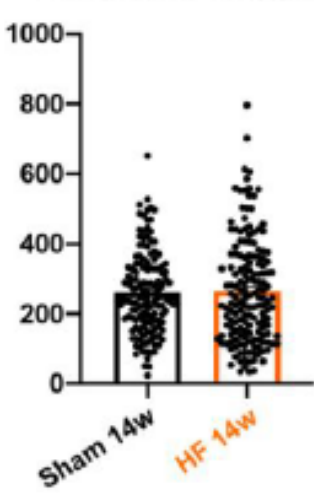

S1BF microglia HF 16w

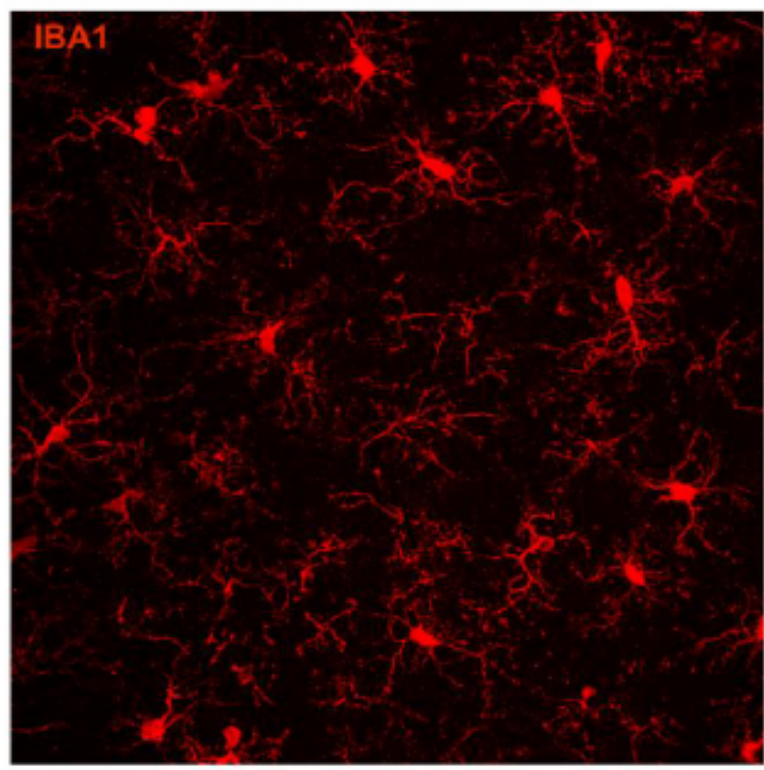

Cell volume

Filament length

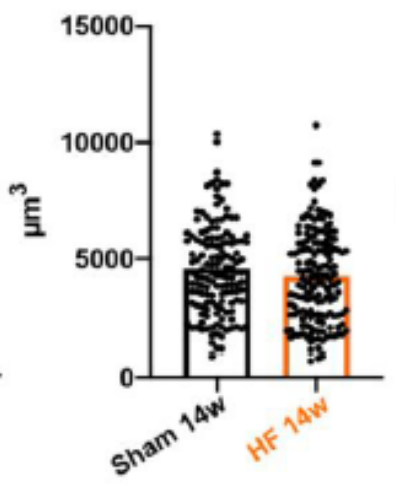

Filament terminals

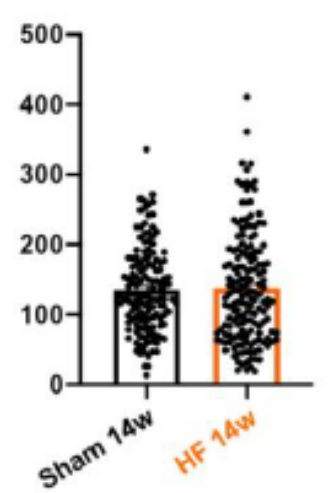

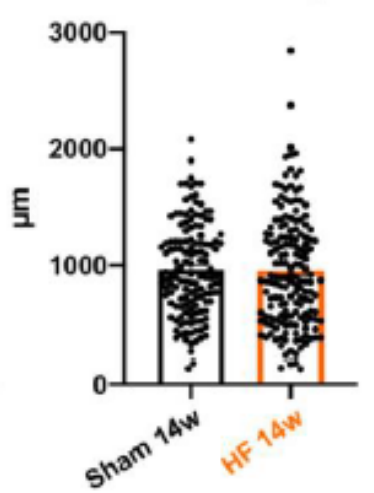

IBA1 intensity

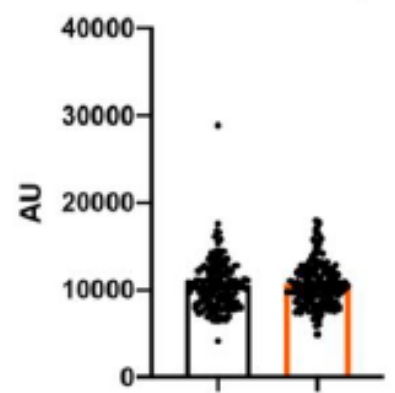

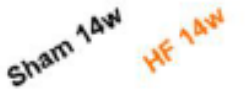

Figure 5 
HF does not induce morphometric changes in somatosensory cortex 1 barrel field microglia (a) representative confocal images show IBA1-stained microglia in the S1BF of HF and sham rats 16 weeks post-surgery, (b) brain scheme shows the topographic location of S1BF microglia that have been used for the morphometric assessment. The red area highlights the S1BF, (c) dot-plot graphs show the individual values of S1BF microglia for surface area, cell volume, filament length, microglial branches, microglial segments, filament terminals and IBA 1 intensity for sham rats $(n=4)$ and HF rats $(n=4)$. One-way ANOVA was used to analyze the data.

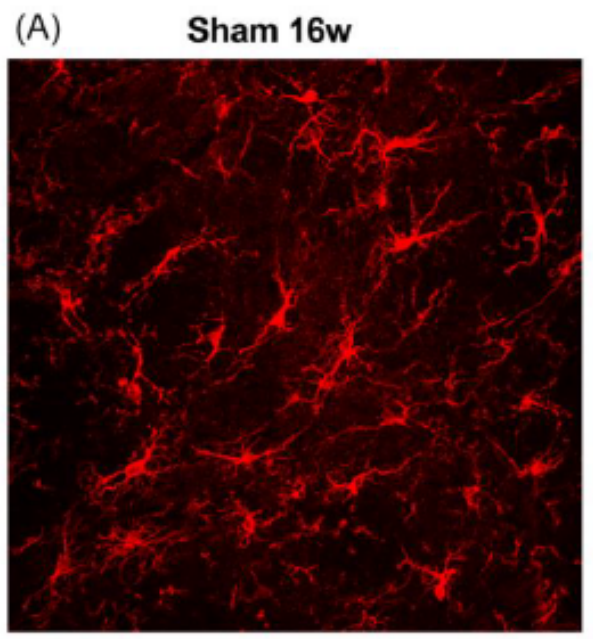

\section{HF surgery $\mathrm{EF}>50 \% 16 \mathrm{w}$}

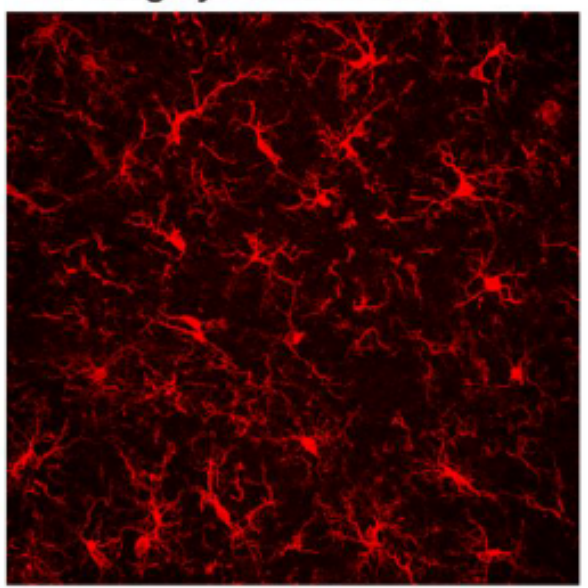

(B)

(C)

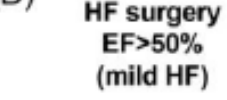

Animals did not develop

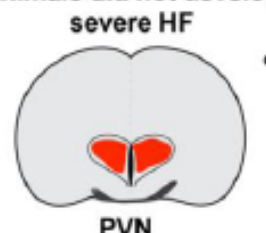

A/P: $-1.1 \mathrm{~mm}$ to $-1.6 \mathrm{~mm}$

Microglial branches

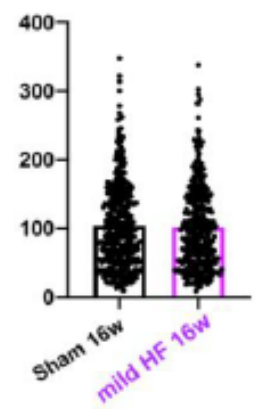

(D)

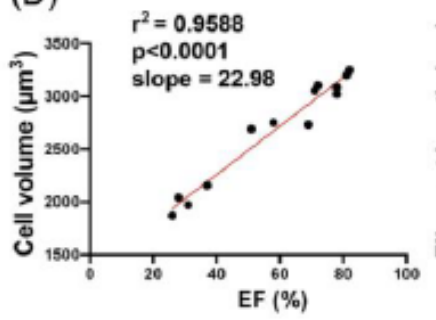

Surface area

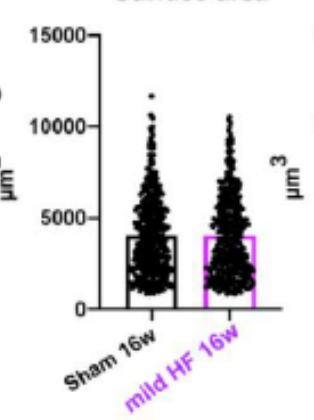

Microglial segments

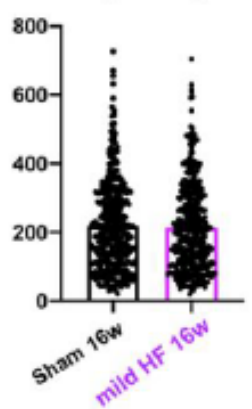

Cell volume

Filament length

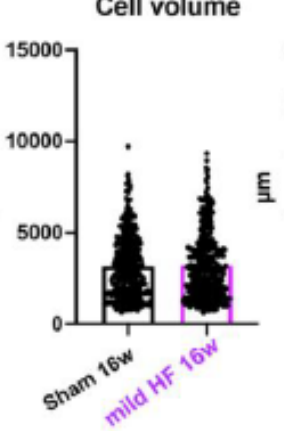

Filament terminals

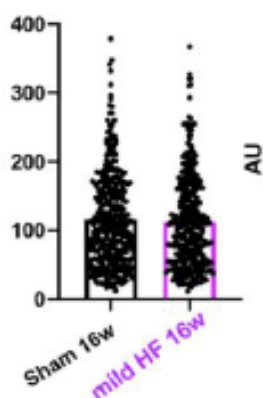

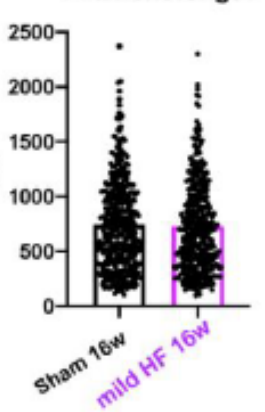

IBA1 intensity

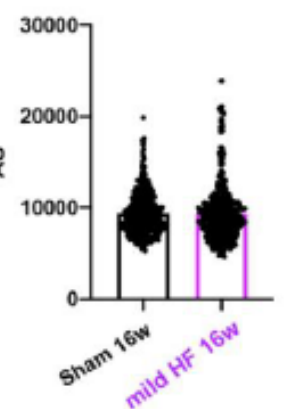

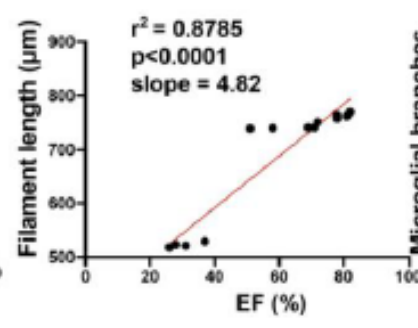

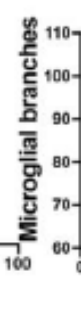

$r^{2}=0.9234$

p $<0.0001$

slope $=0.6199$

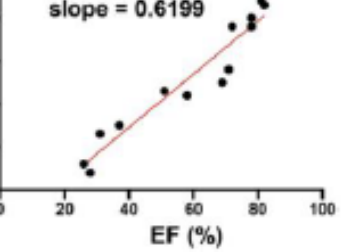




\section{Figure 6}

Morphological changes in microglia are not observed in rats with mild level of HF (a) representative confocal images show IBA1-stained microglia in the PVN of sham and rats that underwent heart failure surgery, but did not develop severe HF ('mild HF'), indicated by an ejection fraction greater than $50 \%$ (EF>50\%), (b) brain scheme shows the topographic location of PVN microglia that have been used for the morphometric assessment. The red area highlights the PVN, (c) dot-plot graphs show the individual values of PVN microglia for surface area, cell volume, filament length, microglial branches, microglial segments, filament terminals and IBA 1 intensity for sham rats $(n=4)$ and mild HF rats $(n=5)$. One-way ANOVA was used to analyze the data, (d) plot graphs depicting cell volume, filament length and number of microglial branches as a function of \%EF values combining sham $(n=4), H F$ rats $(E F<50 \%, n=4)$ and mild $H F$ rats $(E F>50 \%, n=5)$. R2 and $p$ values were obtained following a Pearson correlation analysis. 
(A)

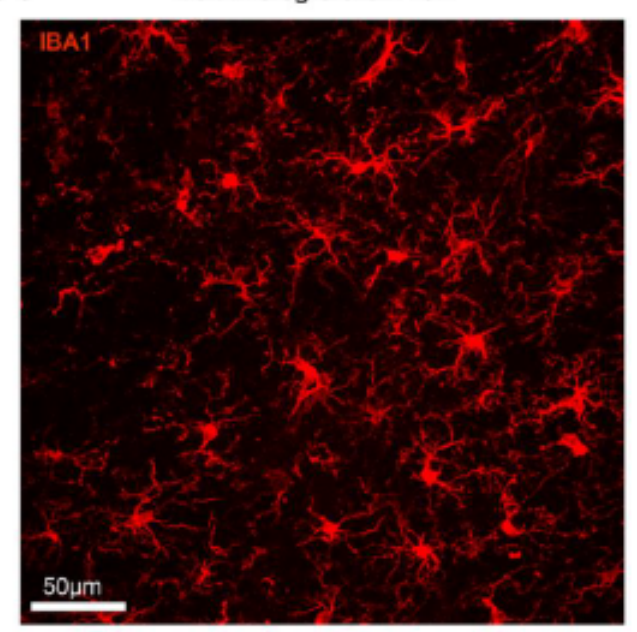

(B)

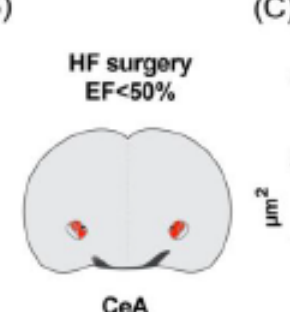

A/P: $-2.0 \mathrm{~mm}$ to $-2.8 \mathrm{~mm}$

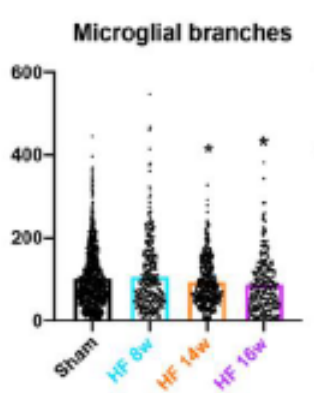

(D)

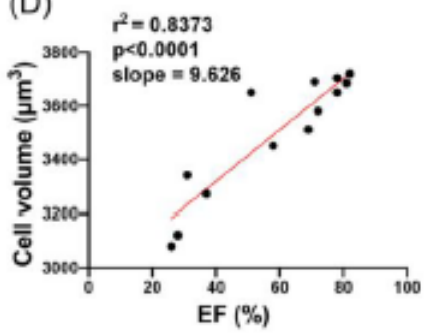

(C)

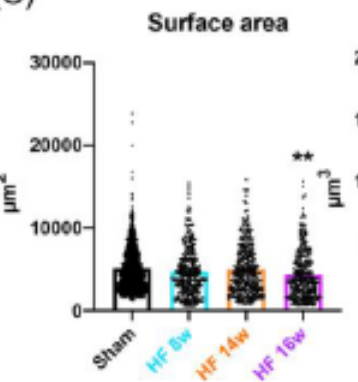

Microglial segments

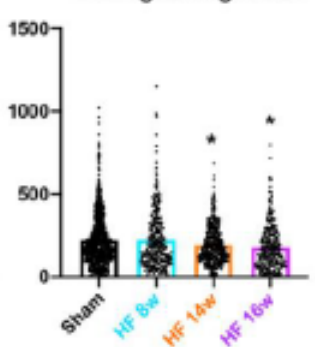

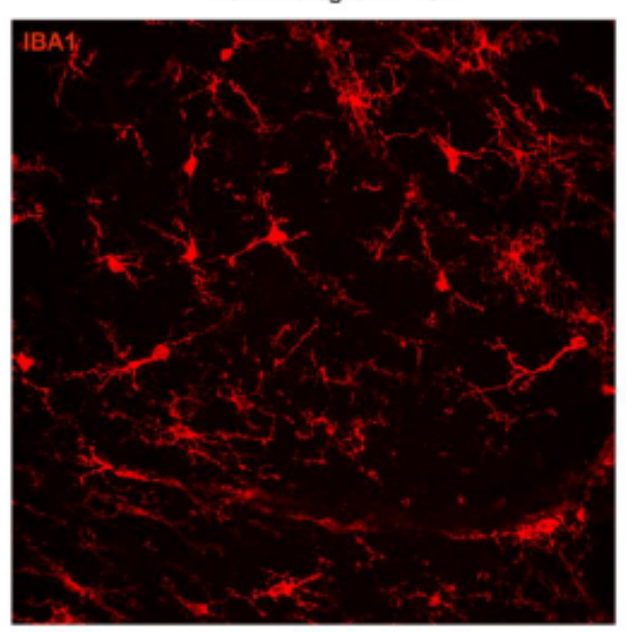

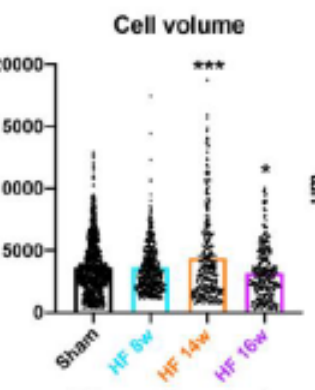

Filament length

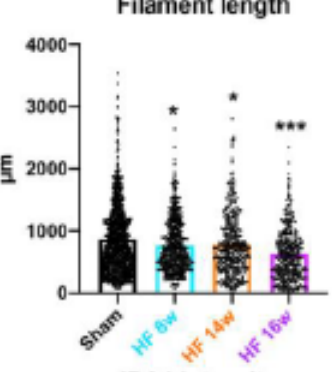

IBA1 intensity
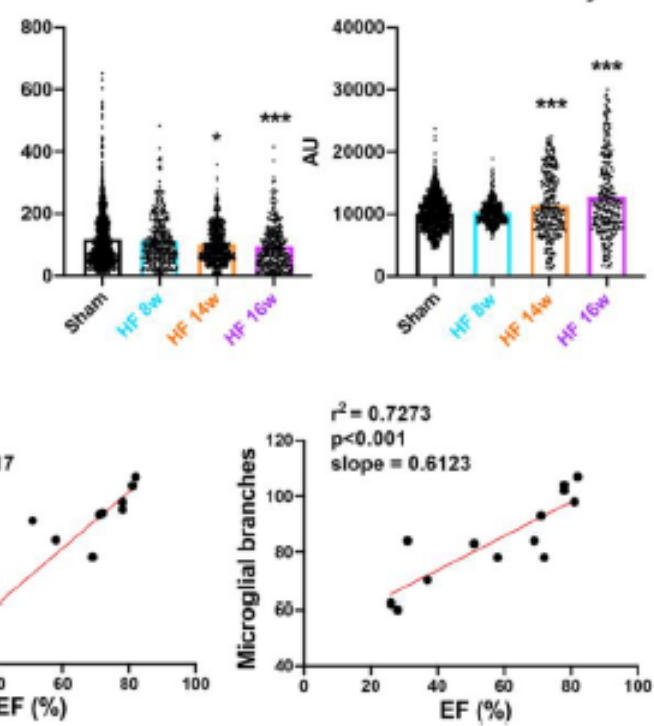

\section{Figure 7}

HF-induced morphometric changes in CeA microglia (a) representative confocal images show IBA1stained microglia in the PVN of HF and sham rats 16 weeks post-surgery, (b) brain scheme shows the topographic location of CeA microglia that have been used for the morphometric assessment. The red dotted line highlights the CeA, (c) dot-plot graphs show the individual values of CeA microglia for surface area, cell volume, filament length, microglial branches, microglial segments, filament terminals and IBA 1 
intensity for sham rats ( $\mathrm{N}=1070$ from 12 rats, pooled) and HF rats at 8, 14 and 16 weeks post-surgery ( $\mathrm{N}=363$ from 4 rats, $\mathrm{N}=355$ from 4 rats and $\mathrm{N}=332$ from 4 rats, respectively). ${ }^{*} \mathrm{p}<0.05,{ }^{* *} \mathrm{p}<0.01$ and ${ }^{* * *} p<0.0001$ vs respective sham, one-way ANOVA followed by Tukey post-hoc test, (d) plot graphs depicting cell volume, filament length and number of microglial branches as a function of \%EF values combining sham $(n=4), H F$ rats $(E F<50 \%, n=4)$ and mild $H F$ rats $(E F>50 \%, n=5)$. R2 and $p$ values were obtained following a Pearson correlation analysis.

(A)

Sholl analysis

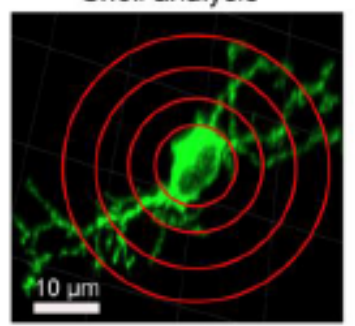

Ramified state High level of complexity
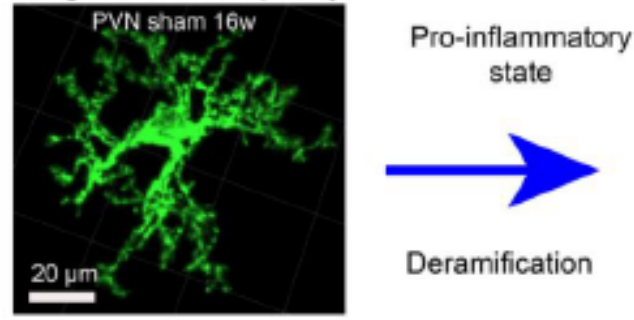

Deramification

(D)

(C)

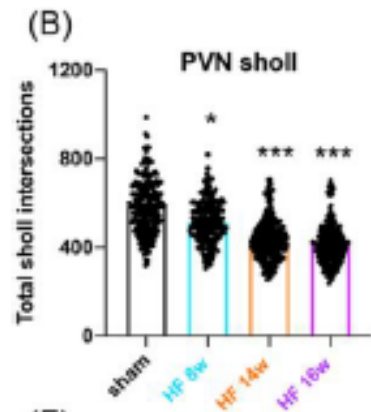

(F)

PVN sholl distribution HF

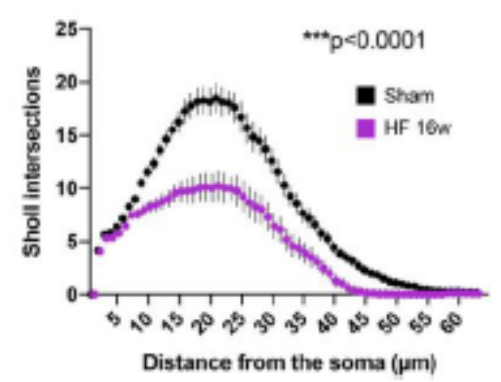

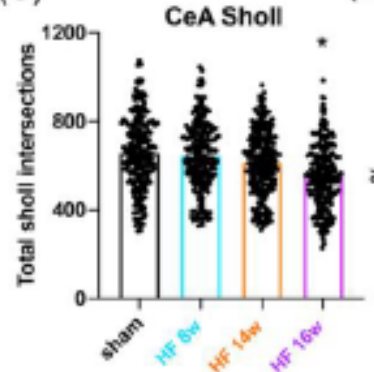

(G)
CeA sholl distribution HF

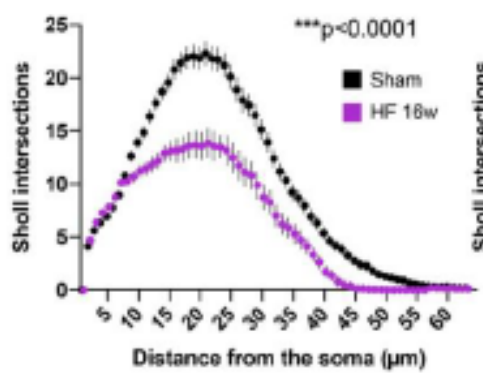

Deramified state

Low level of complexity

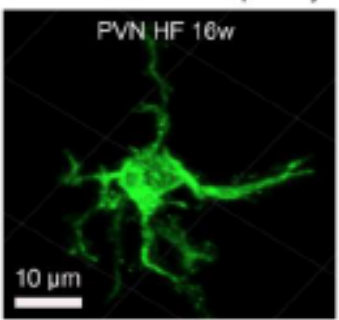

(E)

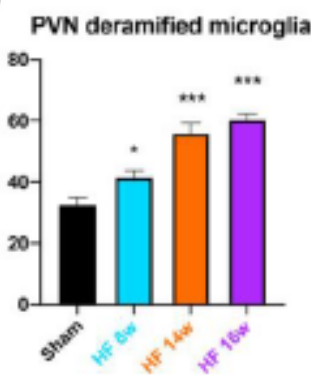

(H)

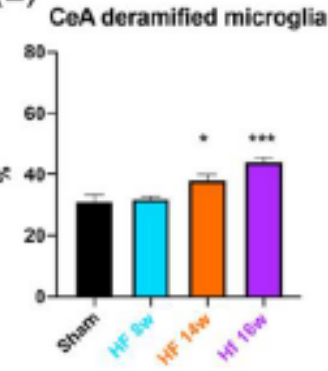

Sholl distribution PVN/CeA

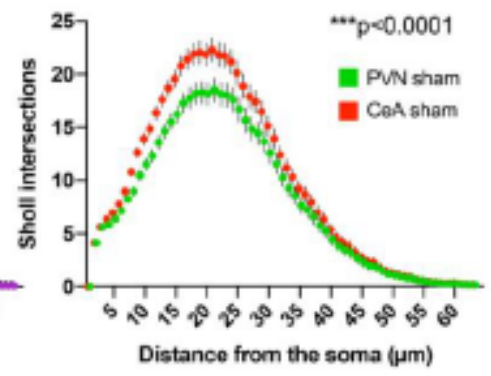

Figure 8

HF induced deramification and increased the proportion of pro-inflammatory microglia in the PVN and CeA (a) scheme depicts the HF-induced transition from ramified to deramified microglia. Red circles depict superimposed spheres centered around microglia somata used for Sholl analysis, (b, c) bar graphs show the mean number of total of Sholl intersections for PVN and CeA microglia in sham rats $(n=12$, pooled) and HF rats at 8, 14 and 16 weeks post-surgery ( $n=4 /$ group), (d, e) bar graphs show the mean proportion of deramified microglia in the PVN and CeA in sham rats ( $n=12$, pooled) and HF rats at 8,14 and 16 weeks post-surgery ( $n=4 /$ group), ( $f-g)$ mean distribution plots of the number of Sholl intersections as a function of the distance from the microglial soma for sham and HF rats 16 weeks post-surgery 
( $n=4 /$ group), (h) distribution plot comparing Sholl intersections as a function of the distance from the microglial soma for PVN and CeA microglia ( $n=12$ /group, pooled). ${ }^{*} p<0.05,{ }^{* *} p<0.01$ and ${ }^{* \star *} p<0.0001$ vs. respective sham, two-way ANOVA or one-way ANOVA followed by Tukey post-hoc test.

(A)

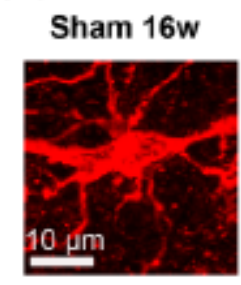

Average soma dameter

$10.4 \mu \mathrm{m} \pm 0.6 \longrightarrow 14.3 \pm 1.1 \mu \mathrm{m}$

Average soma volume

$553.7 \pm 382 \mu \mathrm{m}^{3} \longrightarrow 793.3 \pm 51.7 \mu \mathrm{m}^{3}$

(D)

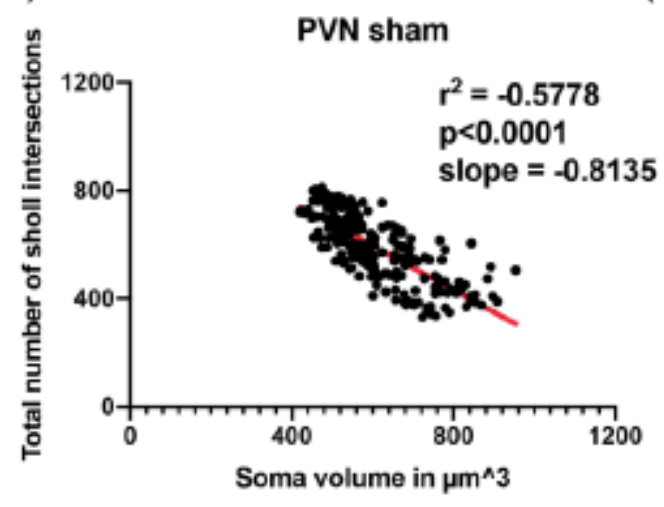

$(\mathrm{F})$

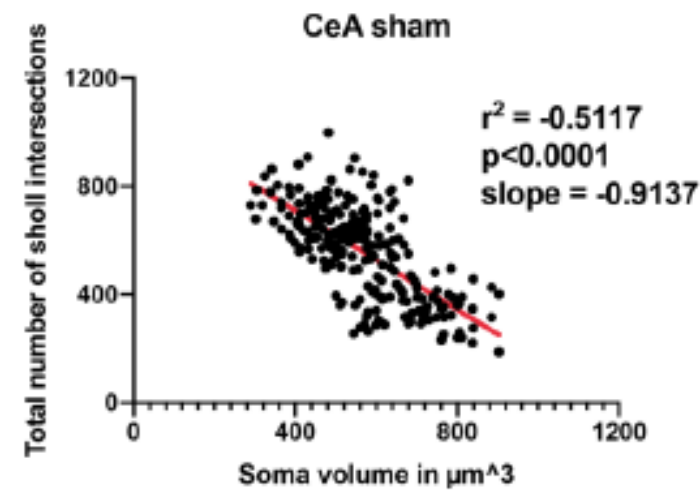

(B)

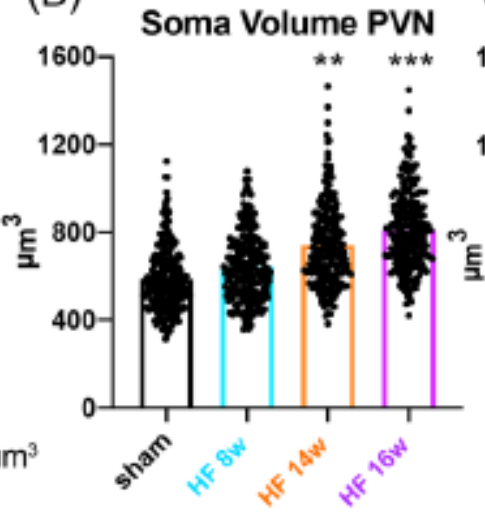

$(\mathrm{E})$
(C) Soma Volume CeA

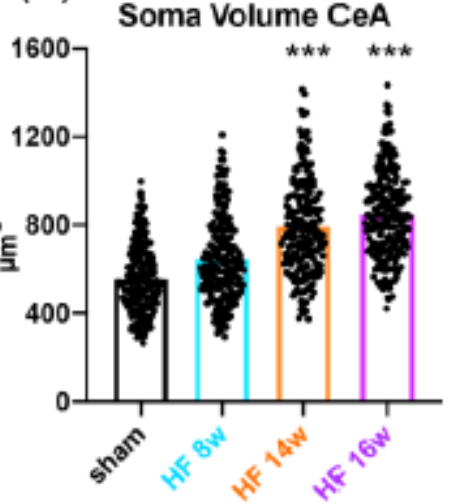

PVN HF

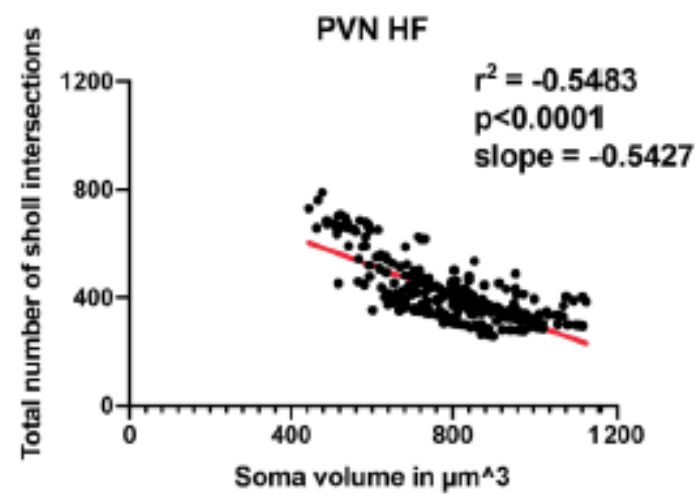

$(\mathrm{G})$

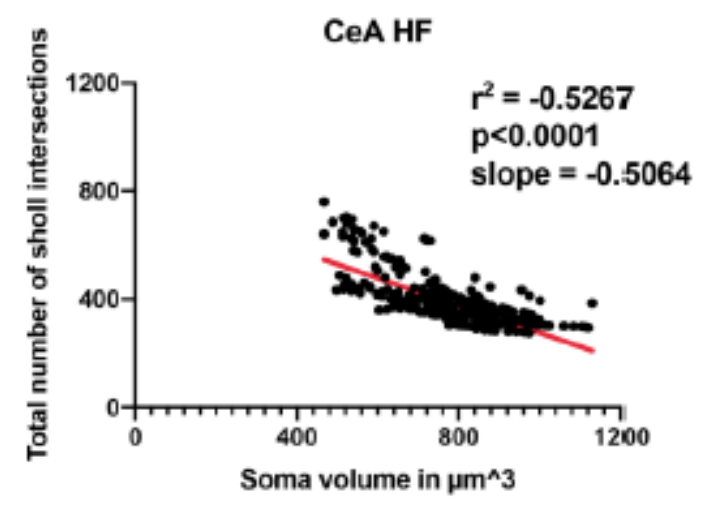

Figure 9

HF-induced somatic swelling of PVN and CeA microglia is correlated with microglial deramification (a) confocal images show a representative example of HF-induced microglial swelling, (b, c) bar graphs show the mean microglia somata volume in the PVN and CeA in sham rats $(n=12$, pooled) and HF rats at 8,14 and 16 weeks post-surgery ( $n=4 /$ group), $(d-g)$ plots showing the total number of Sholl intersections as a function of soma volume for individual microglial cells in the PVN of sham (d) and HF (e) rats and in the CeA of sham ( $\mathrm{f}$ ) and HF (g) rats. Red lines represent best-fit lines assuming a non-linear relationship 
between the total number of Sholl intersections and soma volume. ${ }^{*} p<0.01$ and ${ }^{*} * * p<0.0001$ vs respective sham, one-way ANOVA followed by Tukey post-hoc test or Pearson correlation.
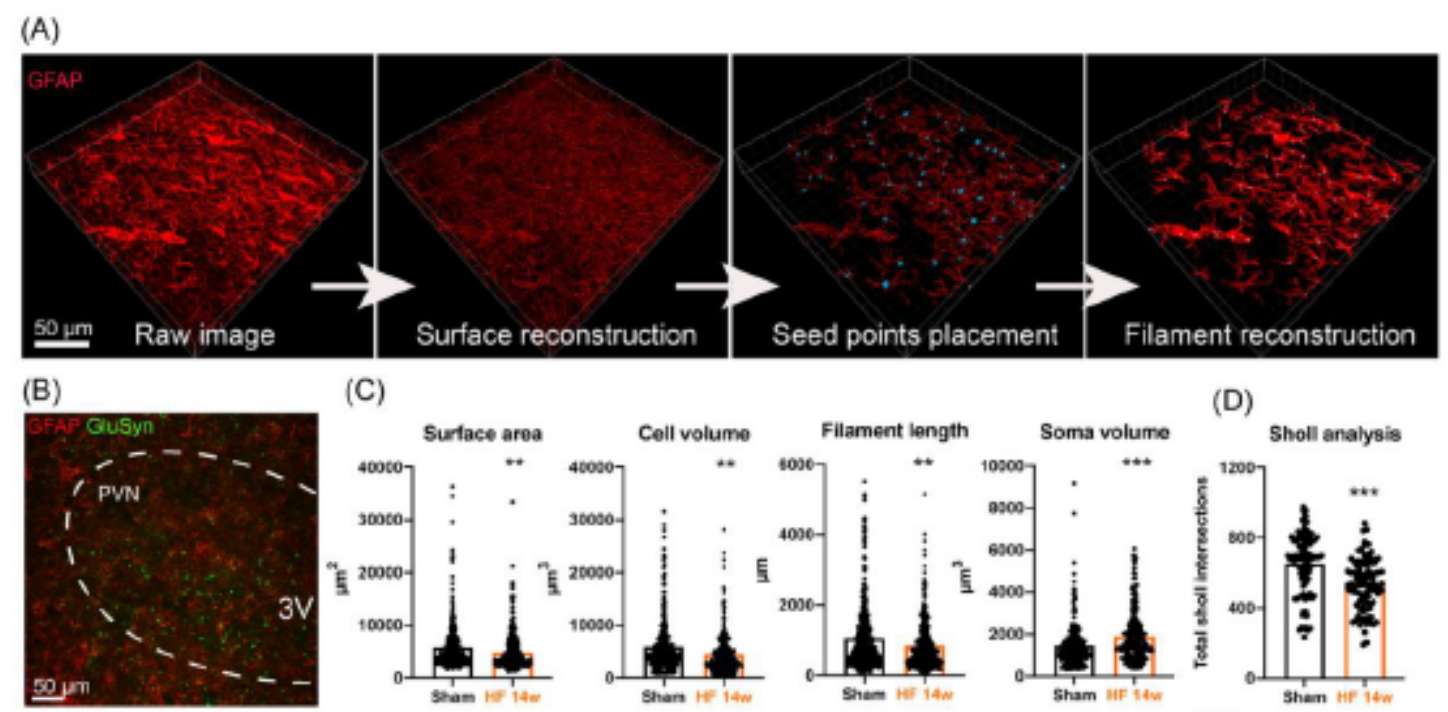

(C)

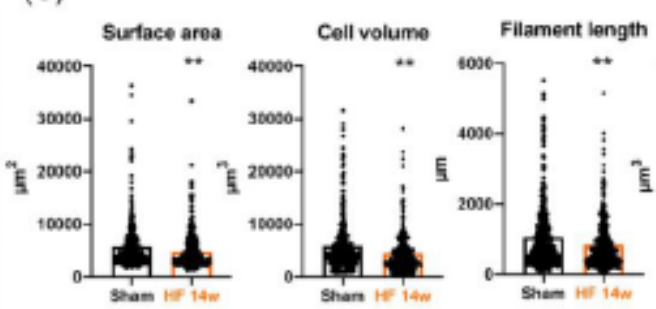

(D)

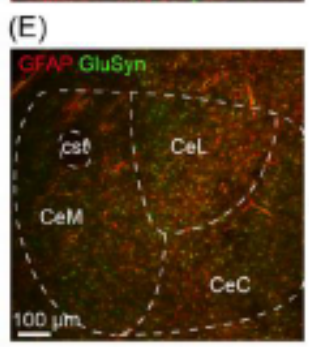

(F)

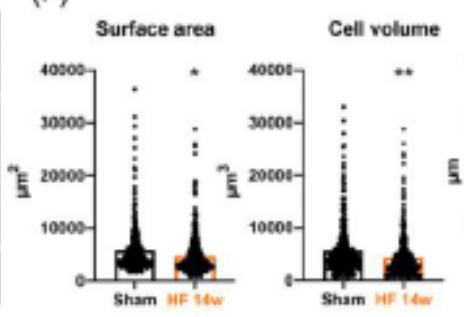

(H)

(I)
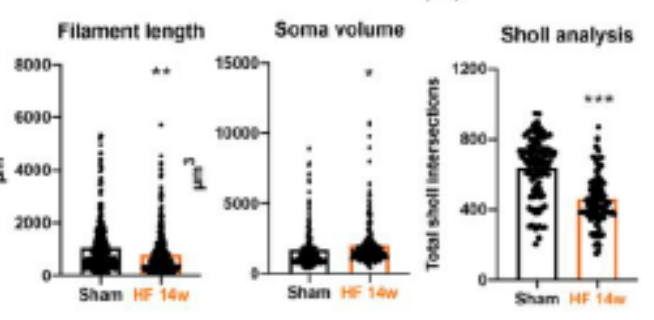

(J)
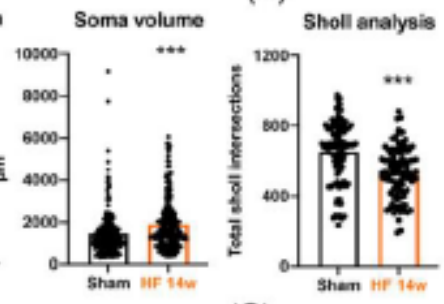

(G)
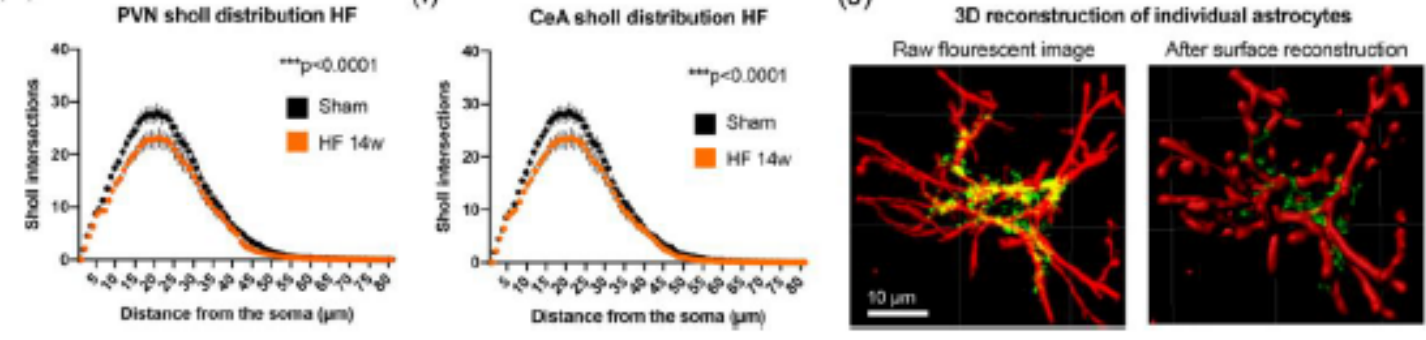

\section{Figure 10}

HF-induced morphometric changes in PVN and CeA astrocytes (a) schematic depiction of sequential steps used for the 3D morphometric reconstruction and assessment of astrocytic processes using IMARIS (GFAP, red), (b) representative confocal image showing PVN astrocytes in a sham rat stained with GFAP (red) and GluSyn (green), (c) dot-plot graphs show the individual values of PVN microglia for surface area, cell volume, filament length and IBA1 intensity in sham $(n=4)$ and HF rats 14 weeks postsurgery $(n=4)$, (d) bar graph shows the mean number of total sholl intersections in PVN astrocytes in sham $(n=4)$ and HF rats 14 weeks post-surgery $(n=4),(e)$ representative confocal image showing CeA astrocytes stained with GFAP (red) and GluSyn (green) in a sham rat, (f) dot-plot graph show the individual values of CeA microglia for surface area, cell volume, filament length and IBA1 intensity in sham $(n=4)$ and HF rats 14 weeks post-surgery $(n=4)(g)$ bar graph shows the mean number of total Sholl 
intersections in CeA astrocytes in sham $(n=4)$ and HF rats 14 weeks post-surgery $(n=4),(h, i)$ mean distribution plots of the number of Sholl intersections as a function of the distance from the microglial soma for sham and HF rats 14 weeks post-surgery ( $n=4 /$ group), (j) confocal images show a single PVN astrocyte from a sham animal before and after surface reconstruction with IMARIS. ${ }^{*} p<0.05,{ }^{*} p<0.01$ and ${ }^{* *} \mathrm{p}<0.0001 \mathrm{vs}$. respective sham, Student's-t test or two-way ANOVA.
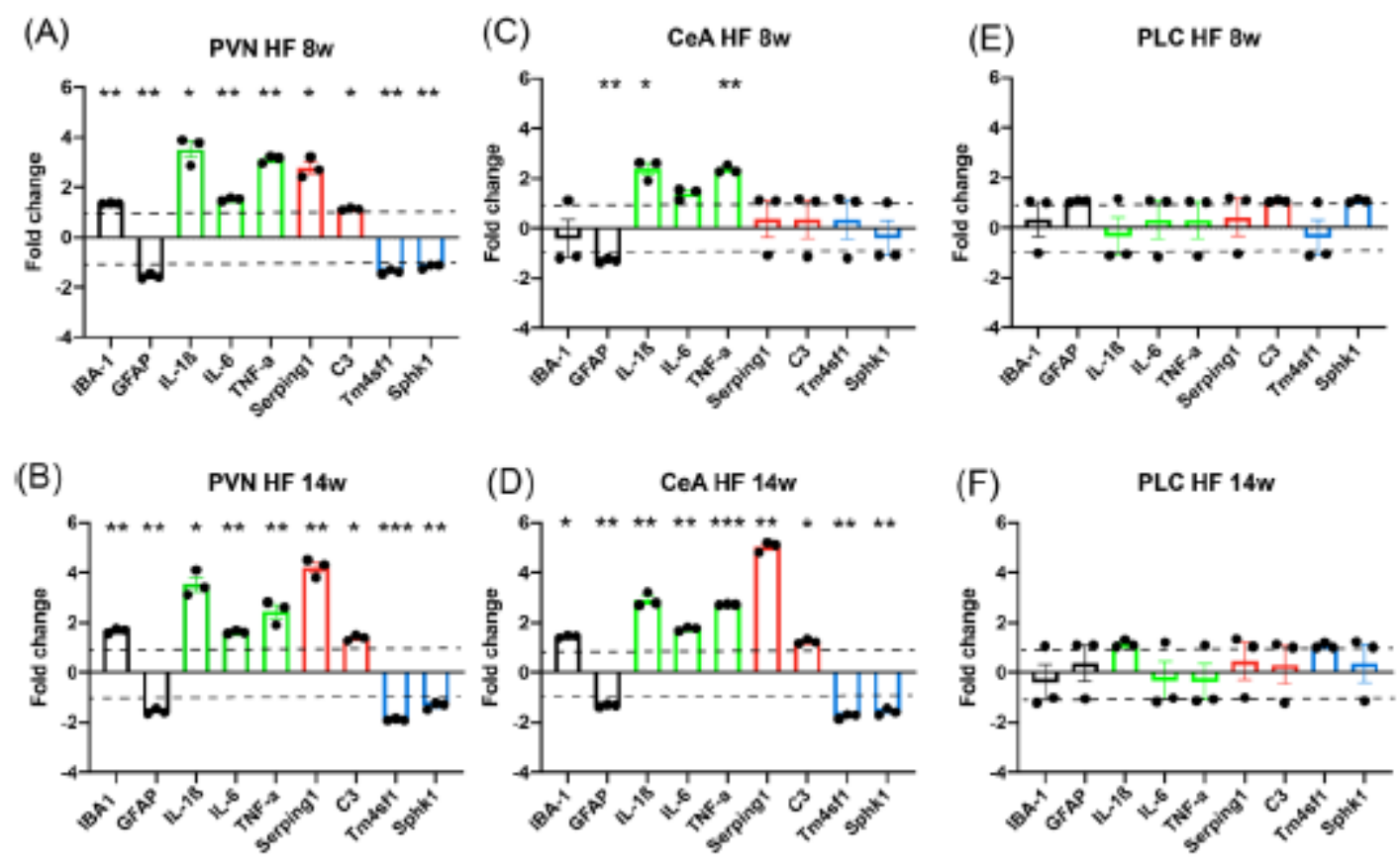

\section{Figure 11}

HF-induced changes in Nl-associated microglia and astrocyte marker mRNA levels (a-f) bar graphs show the mean fold change in mRNA transcript levels in HF rats compared to sham rats in the PVN $(a, b)$, the $\mathrm{CeA}(c, d)$ and the PLC $(e, f)$. All graphs depict the fold changes of mRNA levels in HF group compared to their respective shams. Green bars: cytokines, red bars: a1 astrocyte markers, blue bars: a2 astrocyte markers. Dashed lines indicate the hypothetical mean $(+1 /-1)$ for the one-sample t-test. ${ }^{*} p<0.05,{ }^{* \star} p<0.01$ and ${ }^{* *} p<0.0001$ vs. respective controls, one sample t-test, theoretical mean $=1$. 